\title{
Enantioselective Iodine(I/III) Catalysis in Organic Synthesis
}

\author{
Andrea Flores, ${ }^{\mathrm{a}}$ Eric Cots, ${ }^{\mathrm{a}}$ Julien Bergès, ${ }^{\mathrm{a}}$ and Kilian Muñiz ${ }^{\mathrm{a}, \mathrm{b} *}$ \\ a Institute for Chemical Research of Catalonia (ICIQ), The Barcelona Institute of Science, Av. Països Catalans 16,43007 \\ Tarragona, Spain \\ Fax: +34 977920 224. E-mail: kmuniz@iciq.es \\ b ICEA \\ Passeig Lluís Companys, 23, 08010 Barcelona, Spain
}

\begin{abstract}
Chiral aryliodine(III) reagents have provided an advanced concept for enantioselective synthesis and catalysis. With the advent of chiral iodine(I/III) catalysis, a large number of different structures have been explored in the area. The currently most prominent catalyst design is based on a resorcinol core and the attachment of two lactic side chains bearing ester or amide groups. It enables a privileged modular catalyst synthesis, in which fine-tuning with respect to the specific reaction requirement is straightforward. The present overview summarizes the structural variation and optimization of such chiral aryliodine catalysts and discusses structural properties of the active iodine(III) catalyst states. The status quo of enantioselective iodine(I/III) oxidation catalysis with respect to intramolecular and intermolecular reaction control is reviewed, and specific aspects of the individual catalytic cycles are discussed.
\end{abstract}

\section{Introduction}

Organic chemistry relies on homogeneous asymmetric catalysis as an important working horse for the production of single enantiomers, which have a major impact in biomedical and pharmaceutical sciences. Due to this unparalleled importance of enantiomerically pure compounds, conceptually new endeavors to their synthetic availability are of utmost importance and immediate interest. Within this context, the traditional concept of man-made homogeneous oxidation catalysis has been based on the use of redox-active metals. ${ }^{[1]}$ In this area, the identification and development of suitable chiral ligands $s^{[2-4]}$ has played a major role in order to accomplish defined, kinetically dominating reaction pathways involving effective enantiodiscrimination. ${ }^{[4]}$ Such an approach is largely reminiscent of Nature's concept of metalloproteins ${ }^{[5]}$ which have generated a versatile pool of oxygenation reactions. Their scope has recently been significantly expanded through directed evolution. ${ }^{[6]}$ The advent of organocatalysis ${ }^{[7]}$ has provided an additional class of small organic molecules that can be brought into play for homogeneous oxidation. ${ }^{[8]}$ They can provide complementary reactivity pathways with respect to transition metals. Within the class of metal-free small organic oxidants hypervalent iodine reagents have been widely applied in organic synthesis as oxidants
Keywords: Chirality; Enantioselective Synthesis; Homogeneous Catalysis; Iodine; Oxidation by virtue of their low toxicity, commercial availability, high stability towards atmospheric oxygen and moisture, ease of recovery, easy handling and versatile reactivity. ${ }^{[9]}$ With the goal of providing greener synthetic solutions to the field of oxidation reactions, hypervalent iodine compounds have witnessed a massive growth in the $21^{\text {st }}$ century. ${ }^{[9]}$ The accomplishment of rendering them catalytic has broadened their scope further. ${ }^{[10]}$ One of the predominant current aims of these reagents is complementing or replacing heavy-metal catalysts. Iodine oxidants offer a high potential for the improvement of known reactions and the development of new synthetic transformations. Their nature as polyvalent electrophiles and mild oxidants provides is complemented by their environmental benignness that circumvents the problem of toxicity mainly associated to the use of some transition metals. Common successful approaches are based on the use of aryliodine(I) derivatives, which can be readily oxidized to iodine(III) under the required reaction conditions. For enantioselective transformations, the catalyst design strategy is comparable to conventional transition metal catalysis, in which ionic and/or coordinative bonding of ligands is usually optimized with regard to electronic and stereochemical properties (Figure 1). For aryliodines, the chiral arene substituent is covalently bound. 


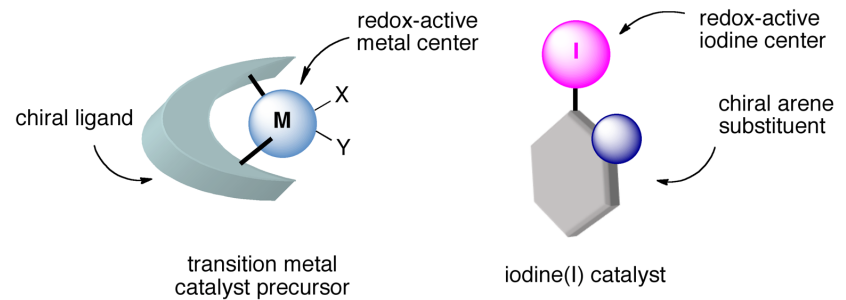

Figure 1. Main concepts for design of homogeneous catalysts: transition metal and aryliodine(III) catalysts.

\section{Chiral Aryliodine Catalysts}

Suitable chiral iodine(III) structures have been identified to a large extent. Following the general advent of iodine(III) reagents as important synthetic tools, enantioselective reaction control has been devised following the principal aspect of introducing chirality elements into the aryl substituent. Their application in enantioselective synthesis has been reviewed previously. ${ }^{[11]}$ Although the resulting chiral reagents have found significant application, the synthesis of preformed iodine(III) centers can in some cases be cumbersome. Clearly, a catalytic reaction course, in which the active iodine(III) is repeatedly generated in situ, represents the superior approach from economic and practicability standpoint. Within this context, non-racemic iodoarenes(I) have been extensively developed since 2008 in order to arrive at homogeneous catalytic performances. With respect to such chiral aryliodine(I) catalysts, all major sources of chirality have been investigated comprising central chirality, axial chirality and helical chirality.

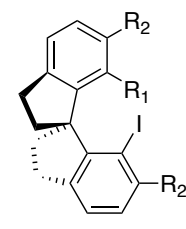<smiles>[R7]c1cccc(Br)c1I</smiles>

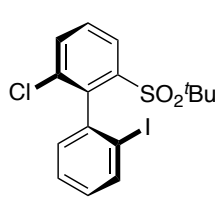<smiles>[R]c1cccc(C(=O)N([R])[2H])c1I</smiles>

6

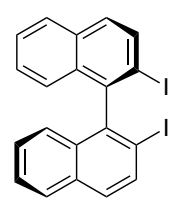<smiles>[R]c1c2c(c(I)c3c1[C@H]1CC[C@H]3C1)[C@H]1CCC[C@H]2C1</smiles>

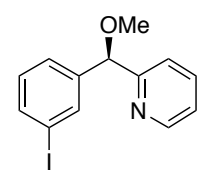

4

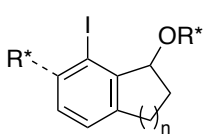

8
Figure 2. Representative structures of chiral aryliodine(I) catalysts.
Andrea Flores was born in 1994 in Cerdanyola del Vallès, Barcelona (Spain). She studied Chemistry at the Autonomous University of Barcelona (Spain), where she obtained her MSc in 2017. She joined the group of Professor Muñiz at ICIQ in the fall of 2018. Her PhD work centers around the development of chiral aryliodine catalysts.

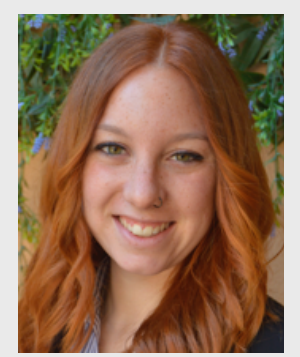

Eric Cots was born in 1994 in Manresa, Barcelona (Spain). After completing his bachelor degree in chemistry at the Institut Químic de Sarrià (URL), he moved to University of Barcelona where he obtained his $\mathrm{MSc}$ in Organic chemistry. Currently he works at ICIQ in Professor Muñiz's group as a $\mathrm{PhD}$ student, where he is developing new reactions with chiral iodine catalysis.

Julien Bergès was born in Toulouse (France) in 1989 and graduated in Chemistry at the Ecole Supérieure de Chimie Organique et Minérale (Compiègne, France). In October 2013 he began his $\mathrm{PhD}$ in the laboratory of Dr. Taillefer at Ecole Nationale Supérieure de Montpellier and received its $\mathrm{PhD}$ degree in 2016 submitting a thesis on the functionalisation of aromatics rings by formation of $\mathrm{C}-\mathrm{C}$ and $\mathrm{C}-\mathrm{N}$ bonds catalysed by copper, iron or under transition metal-free. In July 2017, he joined the group of Professor Muñiz at ICIQ as postdoctoral researcher. His current research focused on the development of methodologies for intermolecular $\mathrm{C}-\mathrm{H}$ amination by electrophilic halide reagents

Kilian Muñiz was born in 1970 in Hildesheim (Germany). He studied Chemistry at the Universities of Hannover (Germany) and Oviedo (Spain) and at Imperial College (UK) and earned his Doctorate in Organic Chemistry for work with Professor Bolm at RWTH Aachen (Germany) in 1998. He carried out postdoctoral research with Professor Noyori at Nagoya

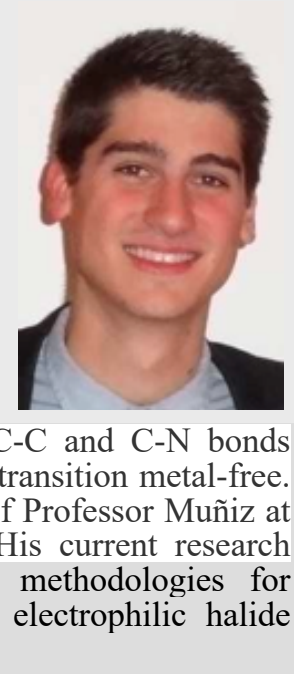

University (Japan), before starting his independent research at Bonn University (Germany) with Professor Dötz (2001-2005). From 2006 to 2009 he held a position as Full Professor at the University of Strasbourg (France), where he was also a member of the Institut Universitaire de France. He moved to Spain in 2009 to join ICIQ in Tarragona (Spain) as a group leader and became ICREA Professor in 2010. His research interests include the 
development of new catalysts for general use in oxidative amination and diamination reactions of hydrocarbons.

One of the first examples of a chiral iodine catalyst 1 was developed by Kita and subsequently extended by Zhang (Figure 2). It contains an electrophilic iodine center and a carbon-based spiro-cyclic backbone with defined chirality. ${ }^{[12]}$ The privileged $C_{2}$-symmetric biaryl motif has also turned out useful as initially shown for compounds $\mathbf{2}$ and $\mathbf{3}$ by Quideau, followed by further developments from Einhorn and Kita. ${ }^{[13]}$ The stereochemical action of lesser popular $C_{1^{-}}$ symmetric structures has also been explored in the architecture of iodoarenes and remains one of the most used to the day for aryliodine catalysts. These catalysts can contain one or more pre-installed chiral centers, and their synthesis has been accomplished through enantiopure starting materials. Different units attached to the iodoarene core such as chiral alkyl substituents, annelated rings or chiral pool-derived groups have thus been effective for the design of catalysts such as 4-8 for a wide range of transformations. ${ }^{[14]}$ As a common feature, the sources of stereochemical information are commonly attached in the proximity of the iodine, thus placing them at the 2- or 2,6-positions of the iodoarene core. This concept was later applied further in the synthesis of modular aryliodine with 2,6dioxygenation motif by Ishihara, Muñiz, and other researchers. ${ }^{[12 b, 15]}$ Arguably, the most important feature of a given chiral catalyst rests with a structural composition that allows for its three-dimensional adaption to a given substrate class within the anticipated chemical transformation. Insight by Ishihara and Fujita has identified lactic acid as a suitable chiral pool-derived auxiliary in the design of iodine catalysts. ${ }^{[16]}$ Examples include the diester 9 and the amides $\mathbf{1 0}$ and $\mathbf{1 1}$.

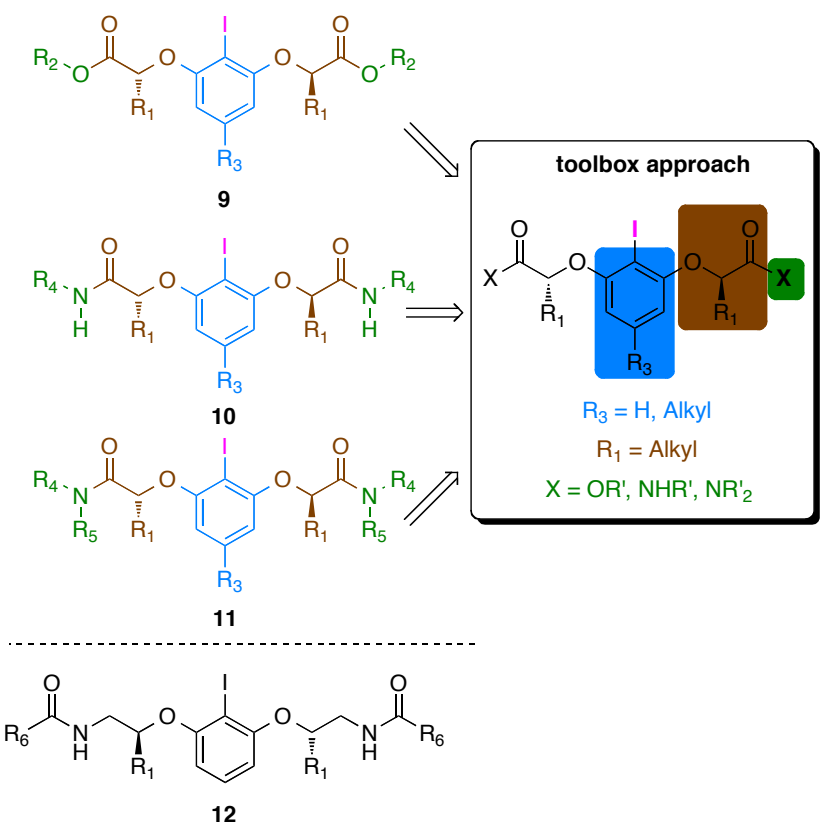

Figure 3. Toolbox approach towards modular iodoarene structures of the resorcinol/lactate nature.

This concept has been proven highly successful due to the fact that structural fine-tuning of the catalysts can be easily accomplished. The underlying modular approach consists in condensation of the corresponding 2-iodo resorcinol cores with lactic acid derivatives and implementation of the ester and amide function. A related conceptual toolbox is based on the functionalization of the central resorcinol moiety with vicinal $\mathrm{N}$-acylated aminoalcohols. Although this approach should provide a similarly attractive entry into catalyst diversification, compounds of type $\mathbf{1 2}$ have so far been explored to a lesser extent.

The major important feature of the resorcinol-derived catalysts rests with the effective formation of helical chirality due to the intramolecular coordination of the chiral side chains. The possibility for the creation of effective chiral environments was first pointed out by Ishihara referring to their potential ability to engage in self $n-\sigma^{*}$ interactions $\mathbf{A}$ between the carbonyl moiety and the iodine (III) formed in situ, as well as Hbonding interaction $\mathbf{B}$ (Figure 4). ${ }^{[15 b, 17]}$

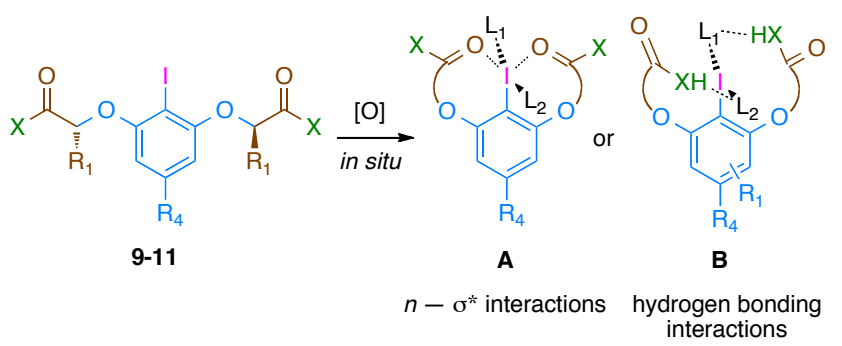

Figure 4. Hypothetical structural arrangements in modular iodoarene-based structures.

Following pioneering insight by Ishihara, ${ }^{[18]}$ this context was indeed confirmed for the resorcinol lactamides 13a,b, which could be oxidized to their corresponding diacetoxy iodine(III) states (Figure 5). ${ }^{[19]}$ X-ray analyses and solution structure determination confirmed the presence of persistent hydrogen bonds between the amide $\mathrm{N}-\mathrm{H}$ and the acetate oxygen atoms at the central iodine. This hydrogen bonding induces a defined supramolecular environment, which is induced by the stereogenic center of the lactamide side chain. The same relative helical chirality was determined for the related secondary amide diacetoxy iodine(III) derivative 14a, for which hydrogen bonding from a water molecule was encountered by X-ray analysis. Formation of supramolecular assemblies may not be limited to intramolecular hydrogen bonding. Work by Hadad and Sunoj using computational chemistry pointed towards a more general helical folding in resorcinol-derived chiral catalyst $\mathbf{1 4 b}$, which is derived from a network of non-covalent attractive interactions. For this prolinyl lactamide-derived bis(trifluoroacetoxy) iodine(III) 
14b, once again the same diastereomeric composition of the helical chirality was determined by computational chemistry suggesting that the lactic stereochemistry is decisive. In this case, a network of attractive noncovalent interactions were detected including the indicated $\mathrm{CH}-\mathrm{FC}$ interactions between methyl ester and methyl groups with the $\mathrm{CF}_{3}$-groups, respectively. ${ }^{[20]}$

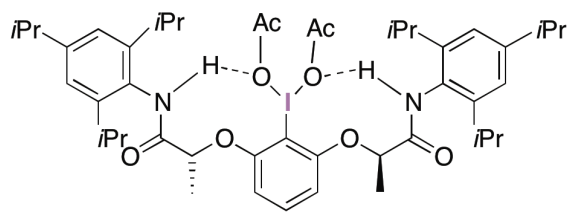

$13 a$

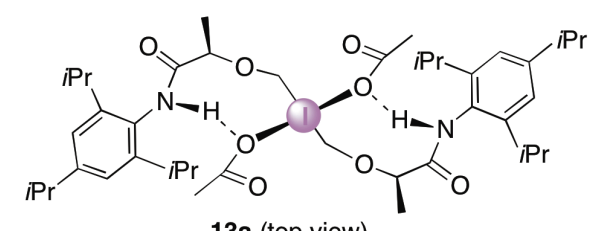

13a (top view)

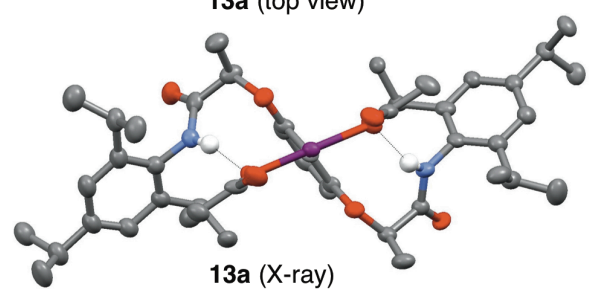

13a (X-ray)
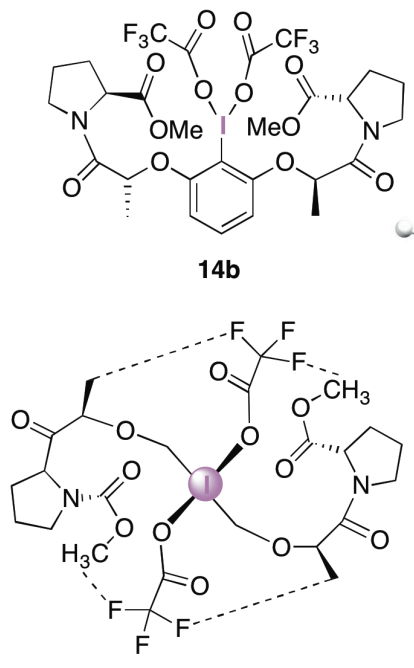

14b (top view)

Figure 5. Structural elucidation of supramolecular assemblies in chiral iodine(III) compounds.

Ishihara had also determined the induction of a related supramolecular helical scaffold by hydrogen bonding for the resorcinol aminoalcohol derivatives 15a and $\mathbf{b}$. For the iodine(III) bismethoxide complex 15a derived from methanolysis of the corresponding bisacetoxy reagent $15 \mathrm{~b}$, X-ray analysis provide unambiguous demonstration of the chiral helical scaffold around the central iodine(III) atom, in which the central chirality<smiles>CCCCNC(=O)C(C)Oc1cccc(O[C@H](C)C(=O)NC(C)C)c1I(OCOCC)OC(C)=O</smiles>

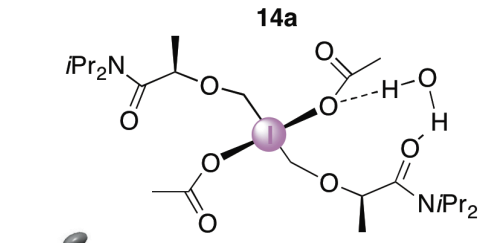

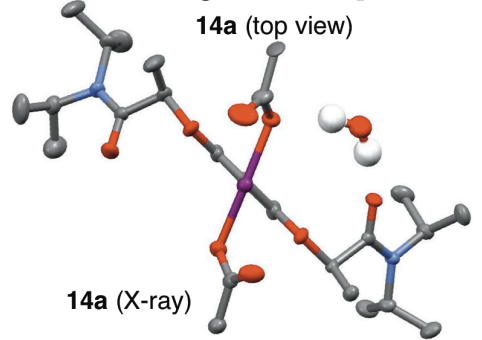

13b (X-ray)

(

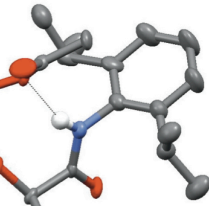

$\underbrace{C}_{C}$

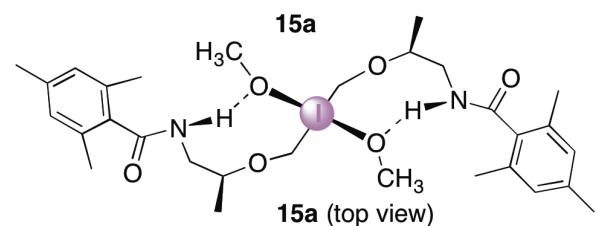

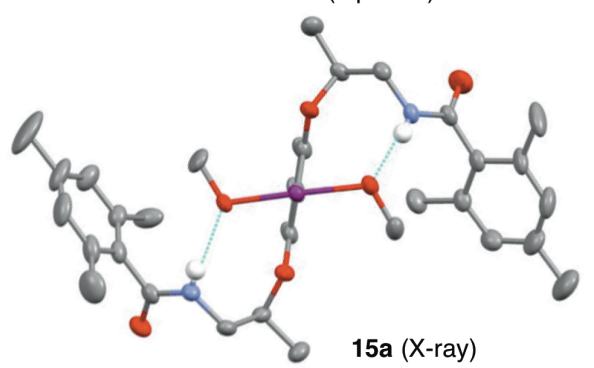

from the aminoether side chains is effectively transferred through hydrogen bonding. Clearly, additional investigation should uncover further aspects regarding potential features for helical structure formation such as the currently elusive $n-\sigma^{*}$ interactions. Such advanced understanding would also provide further detailed design strategies on the working mode of these privileged structures.

In the following, the status quo of iodine(I/III)catalyzed enantioselective oxidation is presented and discussed. 


\section{Enantioselective Catalysis}

\subsection{Catalytic Dearomatization Reactions}

The dearomatization reaction of phenols and derivatives has been widely explored in the last decade. Its potential application in molecular synthesis is the major driving force in the development of new methodologies. Arguably, the oxidative dearomatization of phenols is the most prominent reaction in iodine(III)-mediated chemistry and it has received significant consideration including the development of enantioselective variants..$^{[11 b, 21]}$

The role of chiral iodine(III) reagent has evolved from a stoichiometric variant to the catalytic version, as the latter exhibits much better qualities as explained above. This reaction has been developed in its intra or intermolecular variants, depending on the inclusion or absence of the nucleophile within the substrate structure. Different functional groups regarding the nucleophilic species have been applied including alcohols, acids and amines.

Back in 2008, the first example of a catalytic enantioselective oxidative dearomatization of phenols was accomplished by Kita. In this study, the chiral $\mathrm{C}_{2}-$ symmetric spiro-diiodide 1a was employed as catalyst and the dearomatized product was obtained from the cyclization of the lateral carboxylic acid $\mathbf{1 6}$ upon the $\alpha$-position of the phenol, thus yielding the chiral orthospirolactone structure 17. While the stoichiometric variant of the same transformation yielded up to $86 \%$ $e e$, the catalytic version was slightly less productive (up to $69 \%$ ee, Scheme 1). ${ }^{[22]}$<smiles>[R3]c1cc([R3])c2c(O)c(CCC(=O)O)c([R])c([R])c2c1[13CH2]</smiles>

16

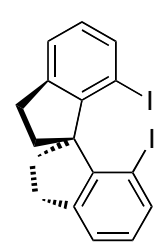

1a $(15 \mathrm{~mol} \%)$ $m \mathrm{CPBA}, \mathrm{AcOH}$ $\mathrm{DCM}, 0^{\circ} \mathrm{C}, 3 \mathrm{~h}$ $16\left(\mathrm{R}_{2}=\mathrm{H}, \mathrm{Br}\right.$,

$\mathrm{R}_{1}=\mathrm{R}_{3}=\mathrm{R}_{4}=\mathrm{R}_{5}=\mathrm{H}$ )

17: $68-70 \%, 65-69 \%$ ee

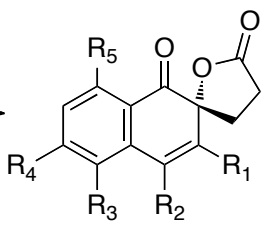

17
Scheme 1. Pioneeering catalytic enantioselective dearomatization of phenols by Kita.

In 2013, Kita performed a significant modification of his oxidative dearomatization, as the introduction of ethyl groups in the ortho positions of the catalyst $\mathbf{1 b}$ permitted access to improved yields and significantly higher enantioselectivities (up to $92 \% e e$ ). ${ }^{[23]}$ The design of the optimized pre-catalyst was achieved by the exploration of the chiral induction through the extension of the equatorial positions next to the iodine center to affect the chiral environment (Scheme 1).

The authors proposed a late stage transition-state model based on the X-ray crystallography of the product 17, in which a ligand exchange with the naphthol oxygen at the formed iodine(III) catalyst $\mathbf{1 8}$ provides the intermediate $\mathbf{C}$. At this stage, the carboxylic acid attacks at the ipso position via the lesshindered $R e$-Face, yielding the observed $R$ enantiomer of the product 17 together with the reduced catalyst containing one iodosoarene moiety (Scheme 2). ${ }^{[23]}$

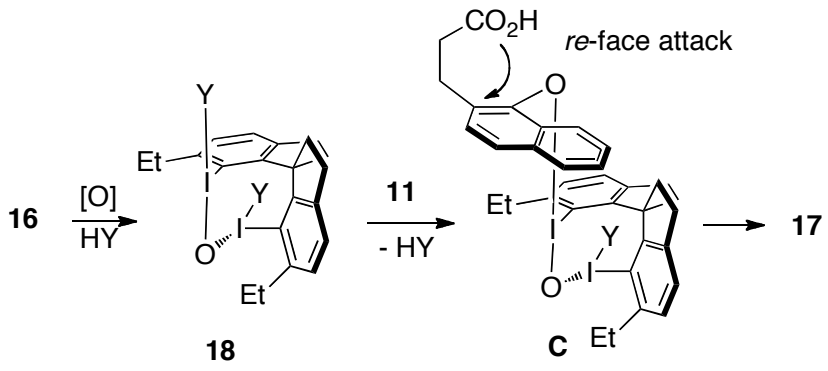

Scheme 2. Transition state proposal for catalyst $\mathbf{1 8}$.

Throughout 2010, Ishihara applied a series of lactatebase chiral aryliodine catalysts $\mathbf{1 0}$ for application in spirolactonization and accomplished notably high yields and enantioselectivities (up to $92 \%$ ee, Scheme 3 ), even for the more reactive class of substituted 1hydroxy-2-naphthalenepropanoic acids. The authors made use of their design motif for the resorcinol bislactamide catalysts discussed above and thereby confirmed the effectiveness of these structures for enantioselective catalysis. ${ }^{[15 b, 17]}$ The depicted derivative 10a represents one of the most common chiral iodine catalysts, and is often referred to as Ishihara's catalyst.
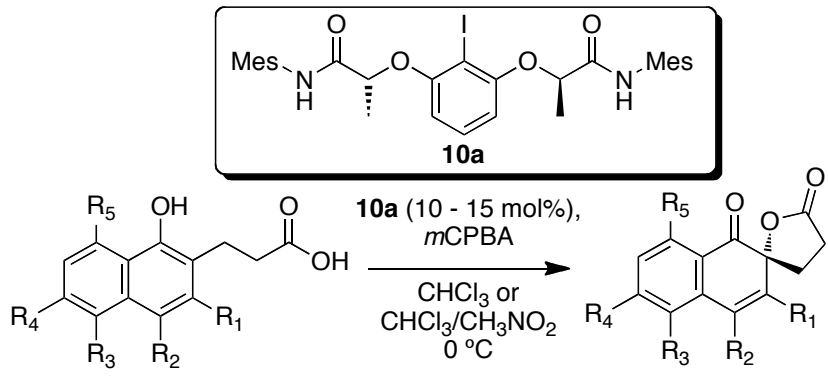

16

$\mathrm{R}_{1}=\mathrm{H}, \mathrm{OMe} ; \mathrm{R}_{2}=\mathrm{H}, \mathrm{Me}, \mathrm{Cl}$

$\mathrm{Br}, \mathrm{Ph}, \mathrm{COAr} ; \mathrm{R}_{3}=\mathrm{H}$, OMe $\mathrm{R}_{4}=\mathrm{H}, \mathrm{OMe}$

$\begin{aligned} & 17 \\ 3 & -94 \%\end{aligned}$

$0-92 \%$ ee

Scheme 3. Oxidative dearomatizative spirolactonization with catalyst 10a. 
Later on, the combination of such intramolecular oxidative dearomatization reactions with a subsequent Diels-Alder reaction were performed as a one-pot domino methodology. A wide variety of lactone products 22 were thus formed in high yields and enantioselectivities from primary dearomatization product 20 and alkenes 21 . The successful catalyst 12a was designed on the basis of the aminoalcohol substitution pattern at resorcinol (Scheme 4). ${ }^{[18]}$

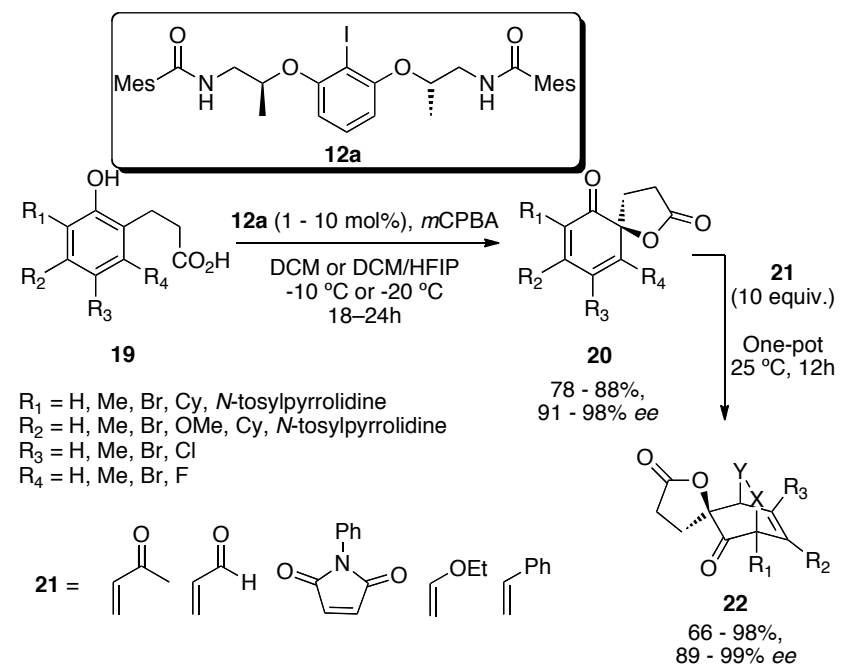

Scheme 4. Domino spirolactonization-Diels Alder reaction with catalyst $\mathbf{1 2 a}$.

Ishihara also observed for some cases that methanol as additive could both accelerate the dearomatization reaction and improve the enantioselectivity. Owing to the capability of the resulting methoxyphenoxy iodine derivative to prevent potential dissociation of the catalyst, the formation of racemic product was greatly suppressed. Recent results from the same group provided a useful methodology for the spirolactonization of 2-naphtol derivatives $\mathbf{2 3}$ in excellent yields and enantioselectivities. In both cases, the chiral resorcinol-derived iodine catalyst 12a makes use of the 2-aminoalcohol concept instead of the common lactate-based scaffold (Scheme 5). ${ }^{[18,24]}$

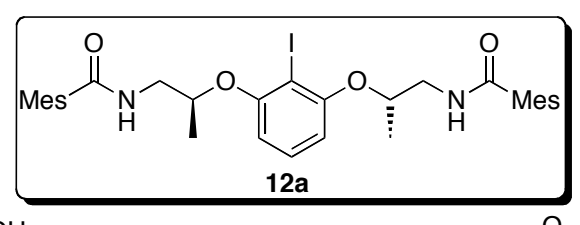

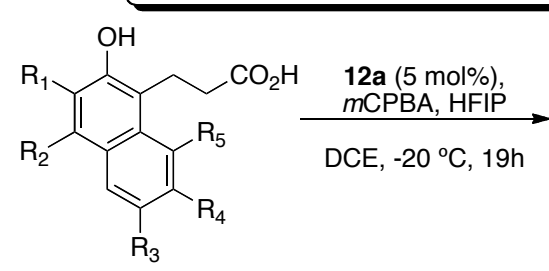

23

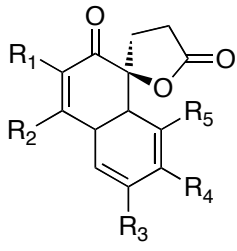

24

$50-99 \%$ $87-99 \%$ ee
$\mathrm{R}_{1}=\mathrm{H}, \mathrm{Br} ; \mathrm{R}_{2}=\mathrm{H}, \mathrm{Me} ; \mathrm{R}_{3}=\mathrm{H}, \mathrm{Br}, \mathrm{CN}$; $\mathrm{R}_{4}=\mathrm{H}, \mathrm{OMe}, \mathrm{OMOM}, \mathrm{OBn} ; \mathrm{R}_{5}=\mathrm{H}, \mathrm{F}$
Scheme 5. Intramolecular dearomatization of 2-naphtol derivatives.

In 2015, Ibrahim proceeded a further broadened contribution to the structural diversification of the spirolactonization, in which a new catalyst motif was introduced. This chiral aryliodine(I) is derived from the octahydrodimethanoanthracene structure, in which the preferred para-methyl substituted derivative 7a yielded a $67 \%$ ee of the desired product 17 (Scheme 6). ${ }^{[25]}$
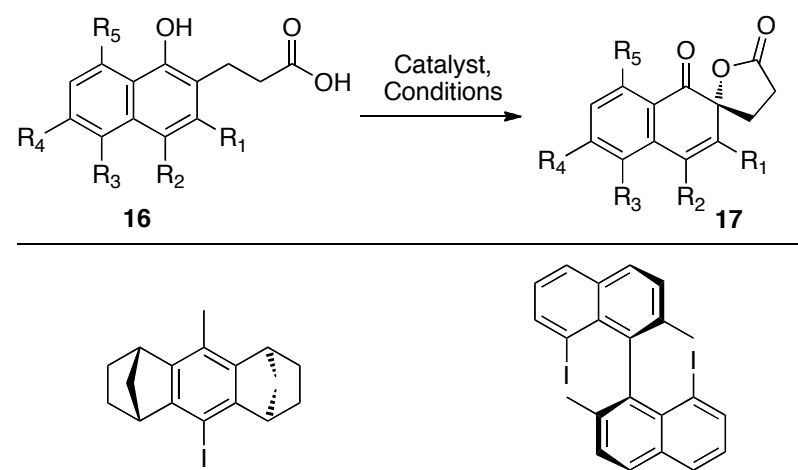

$$
\begin{gathered}
\text { 7a }(10 \mathrm{~mol} \%), \mathrm{mCPBA}, \\
\mathrm{CHCl}_{3}, \mathrm{CHCl}_{3} / \mathrm{CH}_{3} \mathrm{NO}_{2} \text { or } \\
\mathrm{CHCl}_{3} / \mathrm{TFE}, \\
0 \text { or }-20^{\circ} \mathrm{C}, 19 \mathrm{~h} \\
\text { 16: } \mathrm{R}_{1}=\mathrm{R}_{3}=\mathrm{R}_{4}=\mathrm{R}_{5}=\mathrm{H} ; \mathrm{R}_{2}=\mathrm{H}, \\
\mathrm{Br}, \mathrm{Cl}, \mathrm{CN}, \mathrm{Ph} \\
\text { 17: } 36-65 \%, 18-67 \% \text { ee }
\end{gathered}
$$

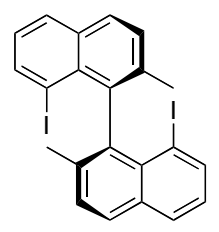
$5 a(10-100 \mathrm{~mol} \%)$, wet $m \mathrm{CPBA}$,
$\mathrm{CHCl}_{3},-40^{\circ} \mathrm{C},<2 \mathrm{~h}$

16: $\mathrm{R}_{1}=\mathrm{H}, \mathrm{Ph} ; \mathrm{R}_{2}=\mathrm{H}, \mathrm{Br}, \mathrm{Cl}, \mathrm{Et}$, $\mathrm{N}_{3}, \mathrm{OBn}$;

$\mathrm{R}_{3}=\mathrm{R}_{5}=\mathrm{H} ; \mathrm{R}_{4}=\mathrm{H}, \mathrm{N}_{3}$ 17: $42-84 \%, 59-78 \%$ ee

Scheme 6. Catalyst exploration for the spirolactonization reaction.

The latest development towards enantioselective dearomatization was provided by Kita in 2017, in which he applied a series of novel catalysts making use of a previously reported binaphthyl 3a. Both a catalytic and stoichiometric amount of the iodoarenes 3a were tested, obtaining up to $64 \%$ ee of the spirolactonized product $\mathbf{1 7}$ for the catalytic variant, similar to the yields and enantioselectivities obtained in the non-catalytic reactions (Scheme 6). ${ }^{[13 c, 26]}$

Ciufolini reported an important expansion of the intramolecular dearomatization reaction, in which the substrates $\mathbf{2 5}$ carried a primary alcohol as nucleophile instead of the commonly employed carboxylic acid. The reaction provided the desired products 26 with high enantioselectivities. Concerning the catalyst, this new dearomatization variant was successful using an Ishihara-type iodoarene 12b. The efforts to accelerate the reaction by a possible Thorpe-Ingold effect due to geminal dimethyl substitution on the quaternary center in $\alpha$-position to the terminal alcohol were unsatisfactory. In addition, a novel aminospirocyclization reaction was reported, in which the analogous heterocyclic products $\mathbf{2 8}$ were formed from 27 in up to $67 \%$ ee (Scheme 7). ${ }^{[27]}$ 

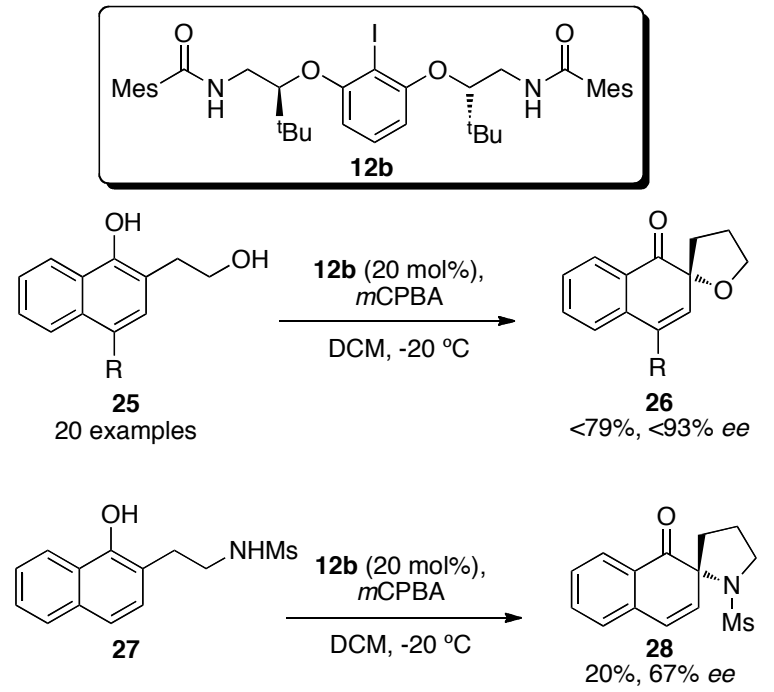

Scheme 7. Catalytic spirolactonization with alcohol and amide nucleophiles.

An additional example by Harned addressed the intramolecular spiroetherification of the parasubstituted phenol derivative 29. The reaction was performed by asymmetric catalysis with the rigid chiral aryliodide 8a incorporating a tartaric bisamide unit. Nevertheless, the yield and enantioselectivity of the reaction remained comparably modest under the explored conditions (Scheme 8). ${ }^{[14 a]}$

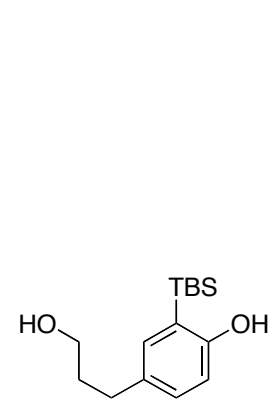

29
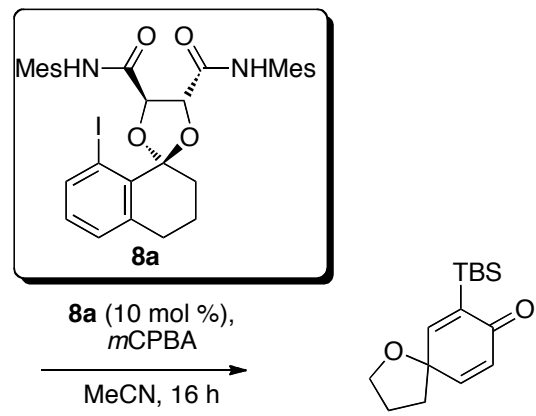

$50 \%, 30: 30$ er
Scheme 8. Harned's catalyst 8a for spiroetherification.

A complementary intramolecular oxidative dearomatization was described by Ishihara. The synthesis of masked ortho- and para-quinones incorporating chiral acetal moieties was accomplished. The reaction with standard catalyst 12a is straightforward for 1,2-dione derivatives $\mathbf{3 2}$ and 34, which were formed with a maximum of $93 \%$ ee (Scheme 9). The larger positional distance of the para-position represented a more challenging transformation with respect to enantioselective face recognition from chiral iodine(III) attachment to the phenol-OH function. To this end, a chiral amide 10b with a significantly increased size of the anilide substituent was designed and the corresponding products $\mathbf{3 6}$ were formed in up to $89 \%$ ee (Scheme 10). ${ }^{[28]}$

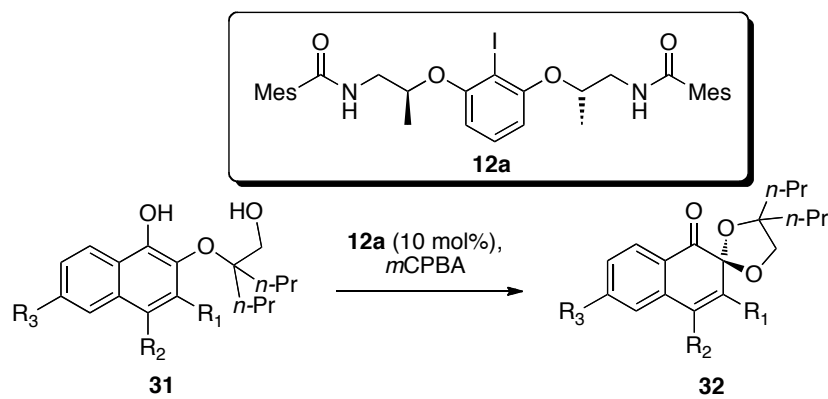

$\mathrm{R}_{1}=\mathrm{H} ; \mathrm{R}_{2}=\mathrm{H}, \mathrm{Cl}, \mathrm{Ph}, \mathrm{Me}$; $\mathrm{R}_{3}=\mathrm{H}, \mathrm{MeO}$

$53-81 \%, 82-93 \%$ ee

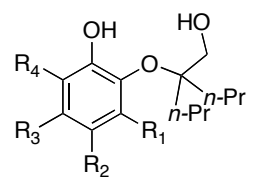

$\mathrm{R}_{1}=\mathrm{H}, \mathrm{Me} ; \mathrm{R}_{2}=\mathrm{H},{ }^{i} \mathrm{Pr}$ $\mathrm{Br}, \mathrm{Cl} ; \mathrm{R}_{3}=\mathrm{H}, \mathrm{Me},\left(\mathrm{CH}_{2}\right)_{4}$ $\mathrm{R}_{4}=\mathrm{H}, \mathrm{Me},\left(\mathrm{CH}_{2}\right)_{4}$

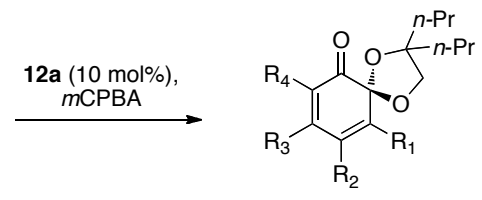

$54-80 \%, 75-91 \%$ ee
Scheme 9. Catalytic synthesis of chiral masked orthoquinones.
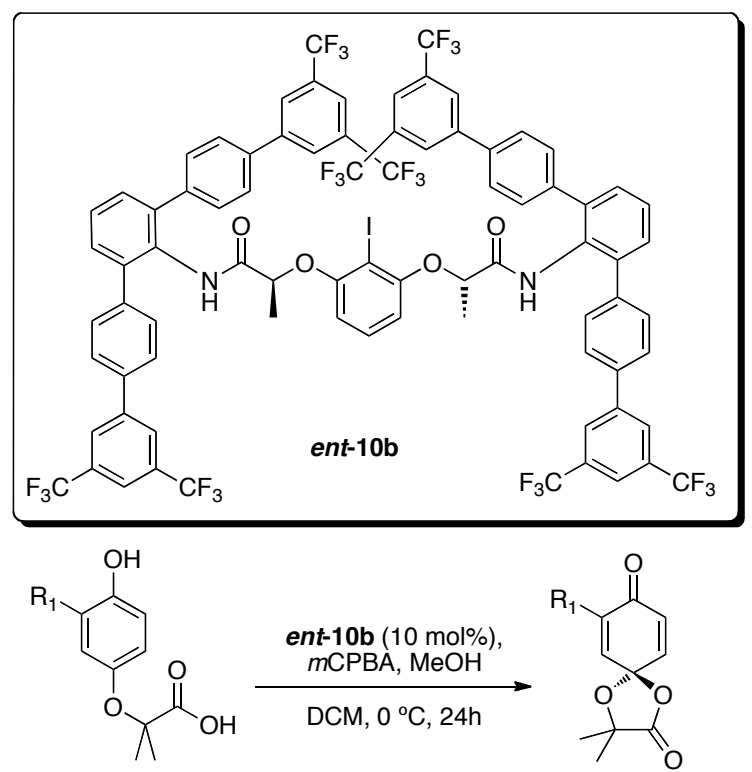

$\mathrm{R}_{1}=\mathrm{Br}$, TBS, TBDPS

$49-87 \%, 10-89 \%$ ee

Scheme 10. Catalytic synthesis of chiral masked paraquinones.

Gong and co-workers developed a new reaction variant, in which an all-carbon stereogenic center was formed throughout the course of dearomatization. In particular, the authors reported the enantioselective spirocyclization of 1-hydroxy-N-aryl-2-naphthamide derivatives 37 enabled by chiral organoiodine catalysis of the chiral resorcinol motif. They presented 12 
different examples $\mathbf{3 8}$ displaying a maximum of up to $92 \%$ ee. The observed beneficial role of the TFE ligand was rationalized by the reduced electron density at iodine and by the resulting diminished dissociation capability of the substrate. Coordinatively saturated iodine(III) leads to the defined substrate-catalyst adduct D (Scheme 11). ${ }^{[29]}$ As to the model for enantiotopic discrimination, cyclization onto the more accessible $\mathrm{Si}$ face of the substrate was the consequence of an effective shielding of the $R e$ face by the proximity of one of the N-phenylamide groups from the side arms of catalyst 10c.

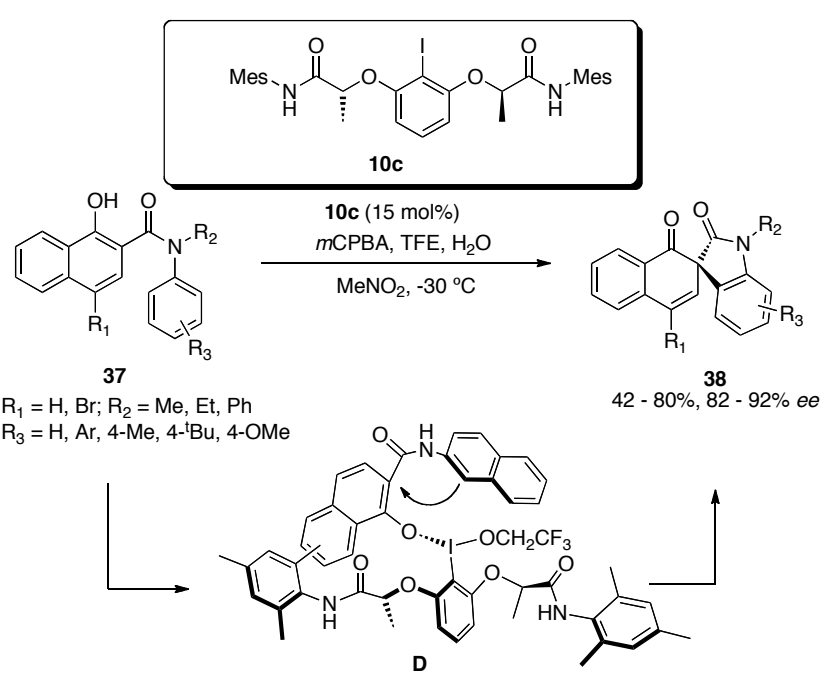

Scheme 11. Formation of an all-carbon stereogenic centre within the dearomatisation reaction.

Additional important recent accomplishments extended the spirolactonization to an intermolecular dearomatization reaction. In this area, Quideau developed the first asymmetric phenol dearomatization by the use of an external nucleophile and chiral iodine catalysts $\mathbf{3 a}, \mathbf{5 b}, \mathbf{5 c}$. They dwelled on the synthesis of either the quinol derivative $\mathbf{4 0}$ or the epoxide-derived molecule $\mathbf{4 1}$, by treating the starting material 39 with a stoichiometric or catalytic amount of the iodoarene, respectively. In this pioneering work, the enantiomeric excess remained moderate with up to $50 \%$ ee for the ortho-quinol 40 and $29 \%$ ee for the ortho-quinol epoxide 41 (Scheme 12). ${ }^{[13 a]}$ Later on, Harned presented a methodology with catalyst 8a compatible with the intermolecular oxidative dearomatisation of phenols 42, which allowed the formation of parahydroxyquinols $\mathbf{4 3}$ with enantioselectivities as high as $60 \%$ ee (Scheme 13). ${ }^{[14 a]}$ The possibility of an alternative catalyst design other than the previleged structures 9-12 was discussed for this reaction, ${ }^{[30]}$ although its realization is still ongoing.
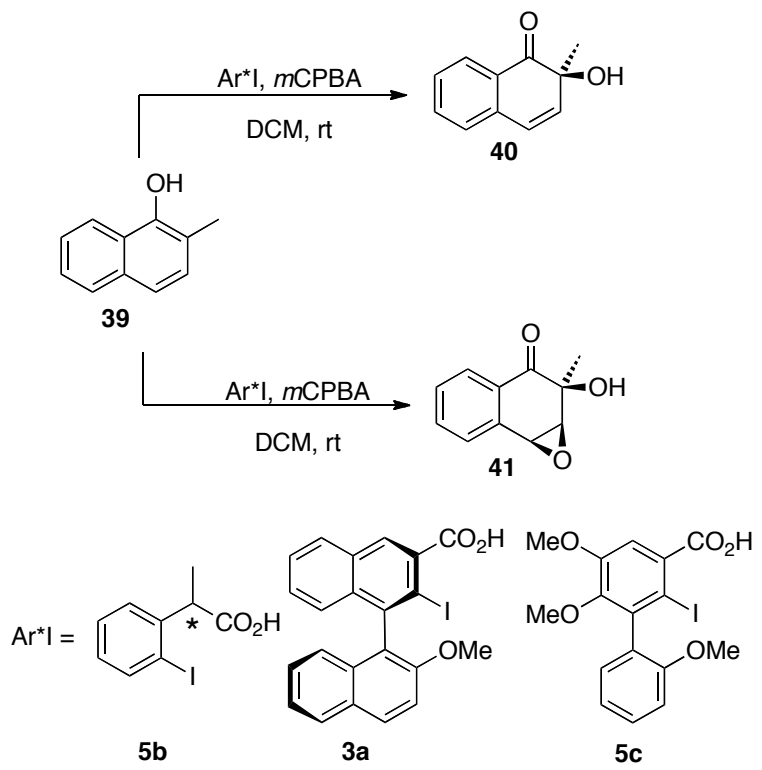

Scheme 12. First iodine-catalyzed enantioselective intermolecular ortho-dearomatisation by Quideau.

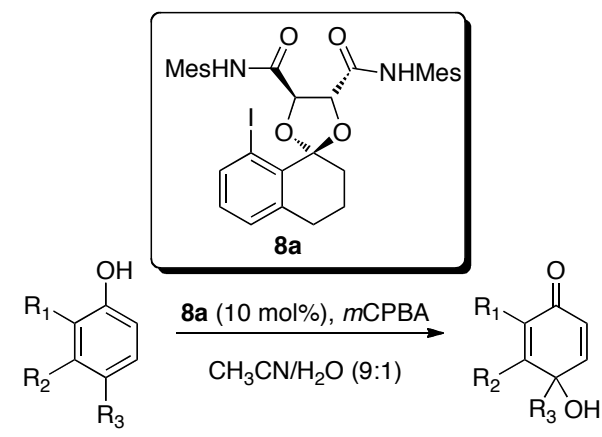

42
$\mathrm{R}_{1}=\mathrm{H}, \mathrm{Me}, \mathrm{Cl}, \mathrm{Br}$,
$\mathrm{TMS}, \mathrm{TBS}, \mathrm{TIPS}$
$\mathrm{R}_{2}=\mathrm{H}, \mathrm{Me}, \mathrm{OMe} ;$
$\mathrm{R}_{3}=\mathrm{Me}, i-\mathrm{Pr}$

$20-79 \%,<60 \%$ ee

Scheme 13. Aryliodine-catalyzed enantioselective intermolecular para-dearomatisation by Harned.

Muñiz elaborated on the previous reaction, specifically for the 2,4-substituted phenols $\mathbf{4 2}$. The use of the modular lactic amide-based iodoarene 10d allowed the obtainment of a substrate range that could be oxidized to the corresponding quinols $\mathbf{4 3}$, some of which showed increased enantioselectivities. Moreover, the reaction scope was broadened to the tosylated aniline analogue $\mathbf{4 4}$, yielding the dearomatized imine product $\mathbf{4 5}$ with up to $34 \%$ ee (Scheme 14). ${ }^{[31]}$ Clearly, these results demonstrate that catalytic intermolecular dearomatizative hydroxylation with synthetically useful enantioselectivities are within reach, and iodine(I/III) catalysis should be the most promising tool for their realization. 


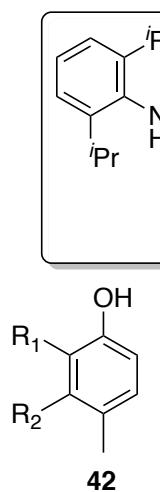

$\mathrm{R}_{1}=\mathrm{H}, \mathrm{Me}, \mathrm{Br}$,

CI, TBS, TIPS, ${ }^{\mathrm{B}} \mathrm{Bu}$

$\mathrm{R}_{2}=\mathrm{H}, \mathrm{Me}$

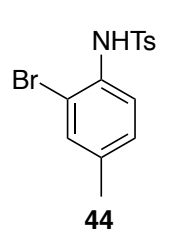

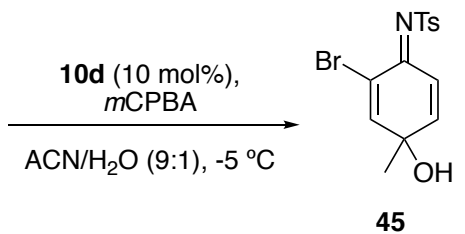

Scheme 14. Aryliodine-catalyzed enantioselective intermolecular para-dearomatisation of phenols and aniline.

Recently, Maruoka designed some additional enantiopure chiral organoiodine catalysts $\mathbf{8 b}, \mathbf{c}$, which are based on the modification of a pivotal chiral hydroxyindanol scaffold (Scheme 15). ${ }^{[32]}$ In line with the general privileged motif, the crucial chiral lactamide substituent at the phenyl ring was maintained. Application of pre-catalysts $\mathbf{8 b , c}$ in the catalytic intermolecular hydrative dearomatization of phenols $\mathbf{4 2}$ to $p$-quinols $\mathbf{4 3}$ allowed for a significant selectivity increase, resulting in up to $84 \%$ ee.

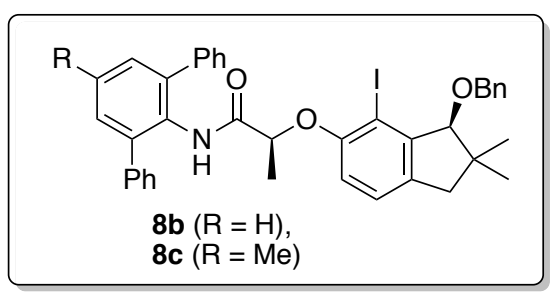<smiles>[R6]c1cc(Br)c(O)cc1Br</smiles>

42

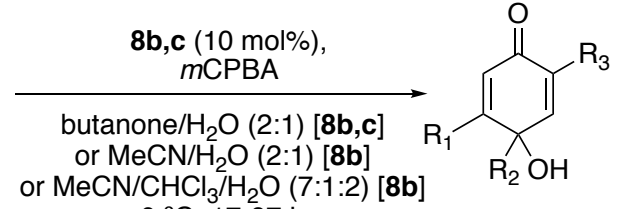

43 $0{ }^{\circ} \mathrm{C}, 17-97 \mathrm{~h}$

$\begin{array}{cc}\text { with } \mathbf{8 b} & \text { with } \mathbf{8 c} \\ 11 \text { examples } & 4 \text { examples } \\ {\left[\mathrm{R}_{1}=\mathrm{H}, \mathrm{Me}, \mathrm{F} ; \mathrm{R}_{2}=\mathrm{Alkyl},\right.} & \left(\mathrm{R}_{1}=\mathrm{H} ; \mathrm{R}_{2}=\mathrm{Me} ; \mathrm{R}_{3}=\mathrm{Cl},\right. \\ \mathrm{R}_{3}=\mathrm{Br}, \mathrm{OTs}, \mathrm{C}_{2} \mathrm{H}_{2} \mathrm{TBDPS}, & \left.\mathrm{F}, \mathrm{TMS}, \mathrm{CH}_{2} \mathrm{OTBDPS}\right) \\ \left.\mathrm{CON}(\mathrm{Bn})_{2}, \mathrm{CON}(\mathrm{MeOMe})\right] & 51-73 \%, 72-84 \% \text { ee }\end{array}$

Scheme 15. Aryliodine-catalyzed enantioselective intermolecular para-dearomatisation by Maruoka.

\subsection{Formal Csp ${ }^{3}$-Functionalization}

The application of iodine(III) catalysis to the asymmetric $\alpha$-functionalization of enolizable carbonyl substrates has emerged as a powerful tool for the stereoselective synthesis of $\alpha$-substituted carbonyl derivatives. Following traditional strategy, ${ }^{[33]}$ this methodology proceeds through an initial enolization of the carbonyl group. After the enantioselective oxidation of the activated alkene by the chiral hypervalent iodine, the reductive substitution of the iodine(III) catalyst allows the insertion of diverse nucleophiles at the $\alpha$-position of the carbonyl. ${ }^{[34]}$ Accordingly, different selective $\alpha$-oxidative $\mathrm{C}-\mathrm{H}$ functionalization reactions (oxygenation, ${ }^{[35]}$ halogenation, ${ }^{[36]}$ amination $^{[37]}$ or fluorination ${ }^{[38]}$ reactions) have been accomplished. The development of suitable enantioselective iodine catalyses has allowed for the introduction of stereogenic centers with defined absolute configuration.

Wirth synthesized various enantiomerically pure iodoarenes $\mathbf{5 d - i}$ that were employed for the lactonization of 5-oxo-5-phenylpentanoic acid 46 with $m \mathrm{CPBA}$ as a stoichiometric oxidant (Scheme 16). Introduction of a weakly coordinating ligand at the iodine(III) species was needed in order to generate the active catalyst and was accomplished by $p$ $\mathrm{TsOH} \cdot \mathrm{H}_{2} \mathrm{O}$. While the yields and $e e$ values of this reaction were moderately high under these conditions, they could be readily applied to an intermolecular $\alpha$ oxytosylation of propiophenones (vide infra, Scheme 18). ${ }^{[15 a]}$

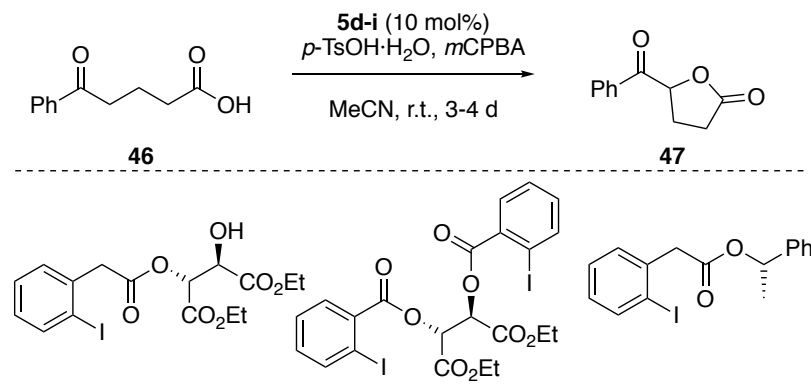

5d, $46 \%, 3 \%$ ee

5e, $28 \%, 3 \%$ ee

5f, $55 \%, 2 \%$ ee<smiles>C[C@H](OC(=O)c1ccccc1I)c1ccccc1Br</smiles><smiles>CC(OC(=O)c1cccc(I)c1)c1ccccc1Br</smiles>

$5 \mathrm{~g}, 67 \%, 2 \%$ ee

5 h, $55 \%, 2 \%$ ee<smiles></smiles>

Scheme 16. Wirth's lactonization of 5-oxo-5phenylpentanoic.

In 2012, Moran and co-workers extended the enantioselective oxidative cyclisation to an $\alpha$ lactonization of 5-oxo-5-phenylpentanoic acid $\mathbf{4 6}$ using chiral aryl iodide $6 \mathbf{a}$ as catalyst. Lactone 47 was formed with $51 \%$ ee, which derives from a remarkably 
high enantiotopic face recognition in this reaction (Scheme 17). ${ }^{[14 c]}$

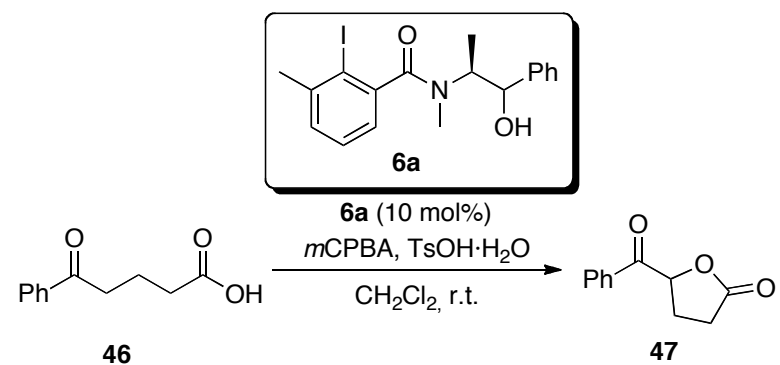

Scheme 17. Aryliodine-catalyzed enantioselective intermolecular para-dearomatisation of phenols and aniline.

Following his pioneering work on the use of preformed chiral iodine(III) reagents ${ }^{[39]}$ in 2007 Wirth presented the first intermolecular $\alpha$-oxytosylation of ketones catalyzed by chiral Koser-type iodoarenes using $m$ CPBA as a stoichiometric co-oxidant, although enantioselectivities remained low (Scheme 18). ${ }^{[15 a]} \mathrm{A}$ higher selectivity for the reaction of propiophenone was achieved by introduction of the ester-based precatalyst $\mathbf{5 k}$. Owing to the presence of the additional chiral menthyl moiety at the ortho position of the aryliodine, an enantiomeric excess of $39 \%$ ee was accomplished. ${ }^{[12 b]}$ In 2010, Wirth designed a series of new chiral iodoarenes, which were also used as catalysts for the enantiomerically enriched $\alpha$ oxytosylation of ketones. The best results were obtained using the bromophenyl-substituted precatalyst 5g. ${ }^{[40]}$ In 2011, the chiral spirobisindane scaffold provided iodoarenes 1 explored as catalysts in the same enantioselective $\alpha$-oxygenation. The resulting Koser-type iodine(III) catalysts were generated using $m \mathrm{CPBA}$. As to an important insight, the authors suspected the acidity of $\mathrm{TsOH}$ to slightly promote product racemization, which led to only moderate enantiomeric excesses below 58\%. ${ }^{[41]}$ In addition, Moran prepared a series of different chiral iodine catalysts through the esterification or amidation of acid chlorides. Use of the best precatalyst $\mathbf{6 b}$ in the $\alpha$ oxytosylation of propiophenone furnished the desired product in $18 \% e e e^{[14 c]}$ Subsequently, Legault and Guibault investigated an activation effect at the iodine(III) catalyst by the introduction of sterically demanding chiral substituents at the ortho position of the aryliodine. In particular, the application of chiral amides, oximes and hydrazones as $\mathrm{sp}^{2}$ Lewis bases was envisioned. Structural analysis and DFT calculations confirmed the observed acceleration to be the consequence of an torsion-induced destabilization within the iodine(III) catalyst. ${ }^{[42]}$ The same group carried out computational investigation to further improve the enantioselectivies. They found out that the most electrophilic dicationic intermediate derived from the oxazoline derivative $\mathbf{5 l}$, in which the oxygen atom chelates the iodine center. ${ }^{[43]}$ In 2013, Einhorn introduced 3,3'-diiodo-BINOL-fused maleimides $\mathbf{5 m}$ as chiral iodine(I) pre-catalysts. The initial focus of their research was put into the optimization of the standard $\alpha$-oxytosylation of propiophenone, which resulted in the choice of a catalyst structure and reaction conditions providing yields and enantioselectivities that are higher than in comparable systems. The authors applied their methodology to different examples giving moderate yields (up to $55 \%$ ) and enantioselectivities of up to $46 \%$ ee) ${ }^{[13 b]}$ The same group further extended their methodology to additional chiral biphenol-based structures, even though the enantioselectivities of the reactions did not notably surpass the ones from previous work. ${ }^{[11 \mathrm{a}]}$ In the present reaction, the low enantioselectivities obtained with catalyst 10a provide a notably exception to its usual superior performance.

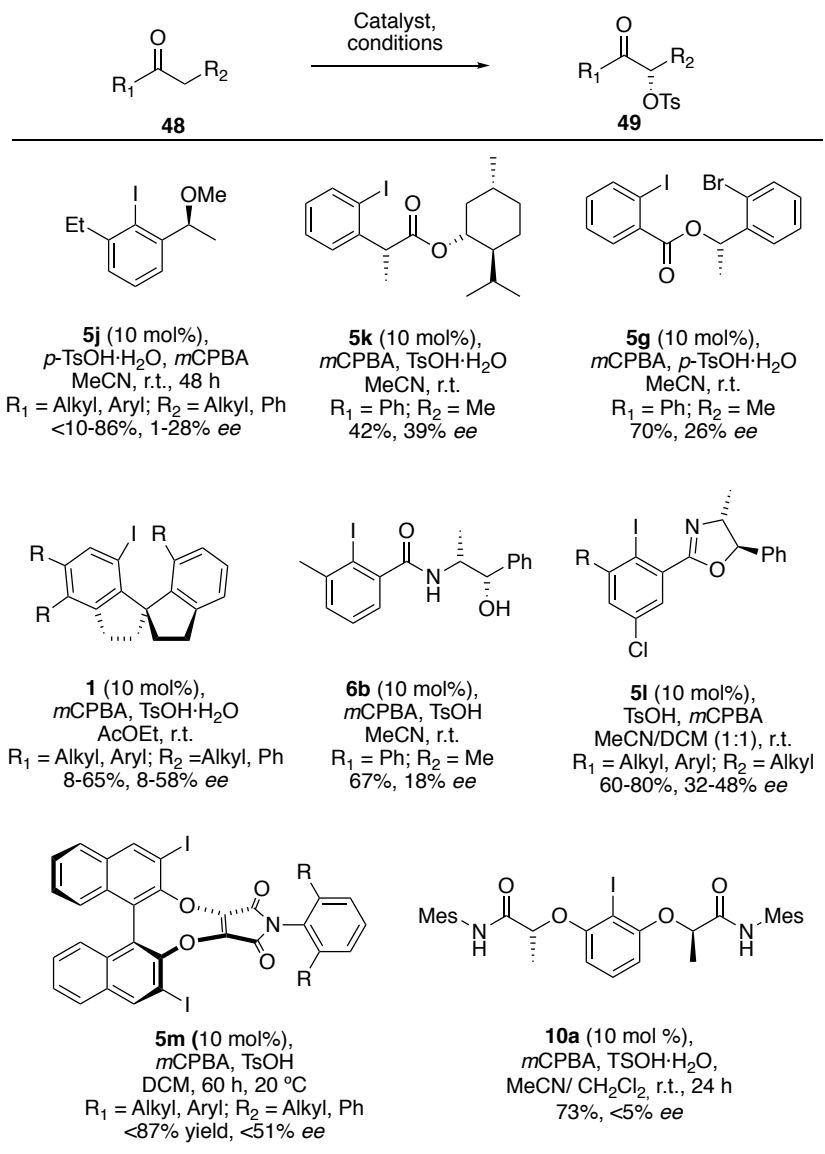

Scheme 18. Aryliodine-catalyzed $\alpha$-oxytosylation of ketones.

The observed difficulties to achieve high enantioselectivities may derive from a mechanistic bias. Within the catalytic cycle, two mechanistically distinct modes of carbon-oxygen bond formation can be considered. The reaction starts with the oxidative conversion of the iodine(I) catalyst to the corresponding chiral Koser derivative. Interaction with the enol tautomer of propiophenone can result in two different substrate-catalyst adducts. In intermediate $\mathbf{F}$, the substrate is C-bound to the chiral iodine(III) moiety, while in intermediate $\mathbf{E}$ an enolate structure 
provides an O-bound iodine(III). For $\mathbf{F}$, direct $\mathrm{S}_{\mathrm{N}} 2$ reaction with tosylate affords the $\alpha$-oxygenated product 49. Alternatively, the reductive displacement of iodine(III) can proceed through a nucleophilic attack onto the $\mathrm{C}-\mathrm{C}$ double bond in intermediate $\mathbf{E}$ yielding the same product. Depending on the exact pathway, the enantioinduction would either be set at the stage of the enol coordination to iodine(III) (intermediate F), or would require an effective shielding of one of the diastereotopic faces of the double bond (intermediate E). The elucidation of C- vs O-bound iodine(III) enolates was recently explored by Norby and Olofsson, ${ }^{[44]}$ and Szpilman ${ }^{[45]}$ and still constitutes an ongoing investigation. Further mechanistic insight should enable the development of effective iodine catalysis for the present reaction.

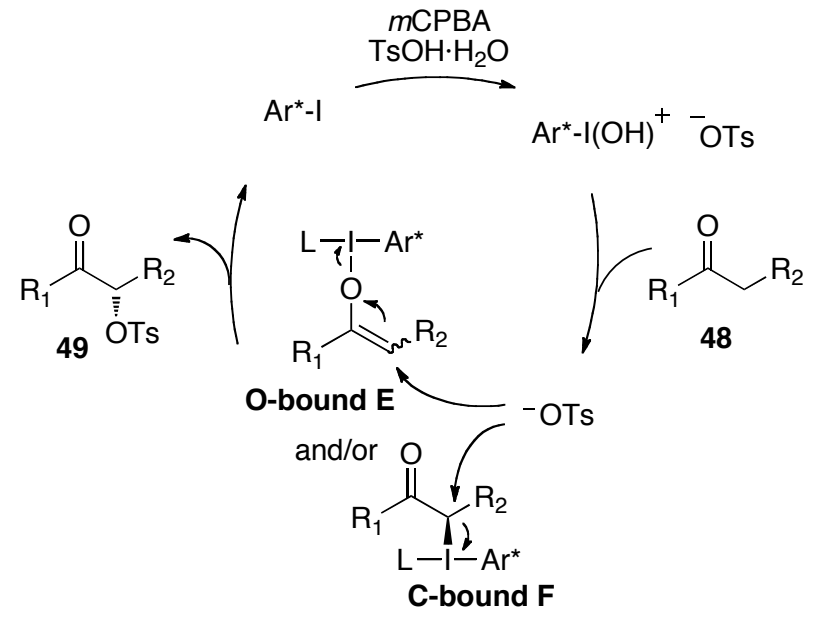

Scheme 19. Proposed mechanisms for the asymmetric $\alpha$ oxytosylation of ketones.

As to a complementary strategy, preformed silyl enolethers and related stabilized enolethers have emerged as useful surrogates. ${ }^{[46]}$ Regarding enantioselective reactions, Legault discovered that the application of the chiral iodine catalyst 10a allowed the formation of the tosylated product 49. While free propiophenone 48 provided almost racemic material, the use of the preformed acetyl enolether 50a under closely related conditions generated a synthetically useful enantiomeric excess (Scheme 20). An even more useful catalytic protocol was developed by the use of the iodine catalyst 11a, which provided 49 with enantiomeric excesses of up to $90 \% .{ }^{[47]}$

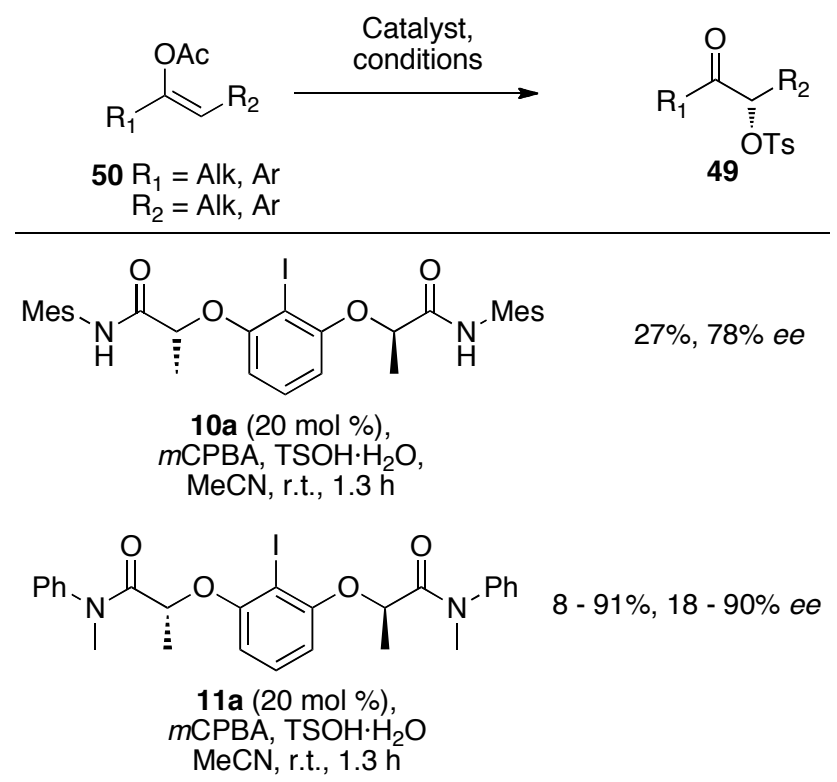

Scheme 20. State-or-the-art aryliodine catalysts for the $\alpha$ oxytosylation of enolethers.

In 2015, an enantioselective catalytic version of the existing $\boldsymbol{\alpha}$-sulfonylation of ketones, ${ }^{[12 a, 35 c, 48]}$ was developed. The reaction used a combination of an axial chiral Koser-type catalyst, $m$ CPBA and free sulfonic acid as combined activator and nucleophile source. The reaction proceeded readily for several sulfonamides providing the $\alpha$-oxygenated products with up to $68 \%$ ee ${ }^{[49]}$ In line with the mechanistic discussion for the parent ketone substrates, the authors suggested the involvement of two different intermediates including an iodine-carbon bound intermediate $\mathbf{F}$ and an O-bound enolate $\mathbf{E}$. The former one would furnish product 51 through an $\mathrm{S}_{\mathrm{N}} 2$ reaction. The latter, which demands an attack at the alkene, was favored and its involvement was corroborated by theoretical investigation (Scheme 21). ${ }^{[50]}$

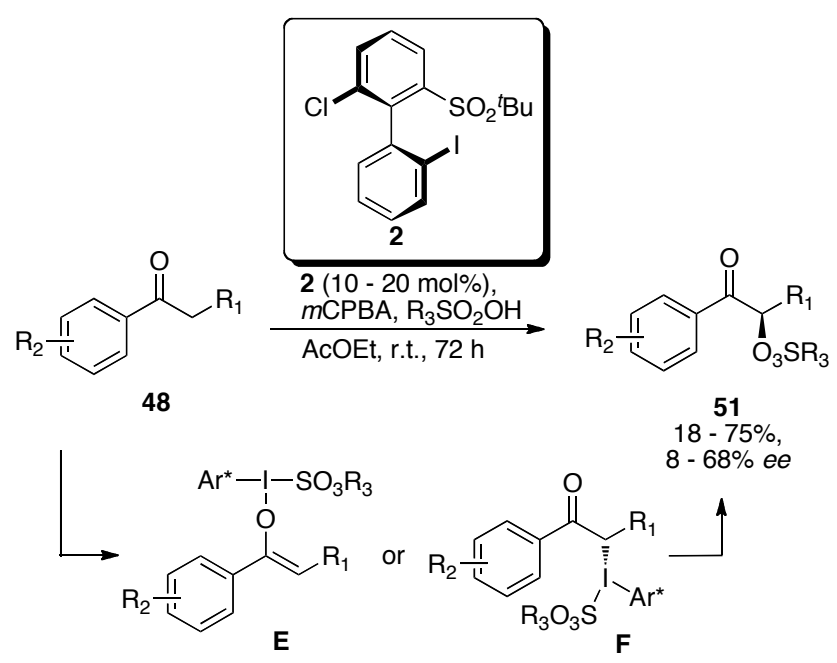

Scheme 21. Enantioselective $\alpha$-sulfonylation of phenones. 
In addition to the oxygenation reaction, Legault obtained the related $\alpha$-chlorinated 1-phenylbutanone from the corresponding chloroalkene precursor using a lactamide derived resorcinol iodine(I) reagent in stoichiometric amounts in combination with $m \mathrm{CPBA}$ and $\mathrm{TsOH}$, and vinyl chloride. This interesting approach provided an enantiomeric excess of $27 \%{ }^{[36]}$ In 2014, new insight on halogenation was reported with the catalytic fluorination of $\beta$-dicarbonyl-type $\mathbf{5 2}$ by use of an efficient combination of chiral iodoarene 3, $m \mathrm{CPBA}$ and HF. Although the reaction was completely selective, the enantioselectivities and yields for the fluorinated products $\mathbf{5 3}$ remained moderate (Scheme 22). ${ }^{[51]}$

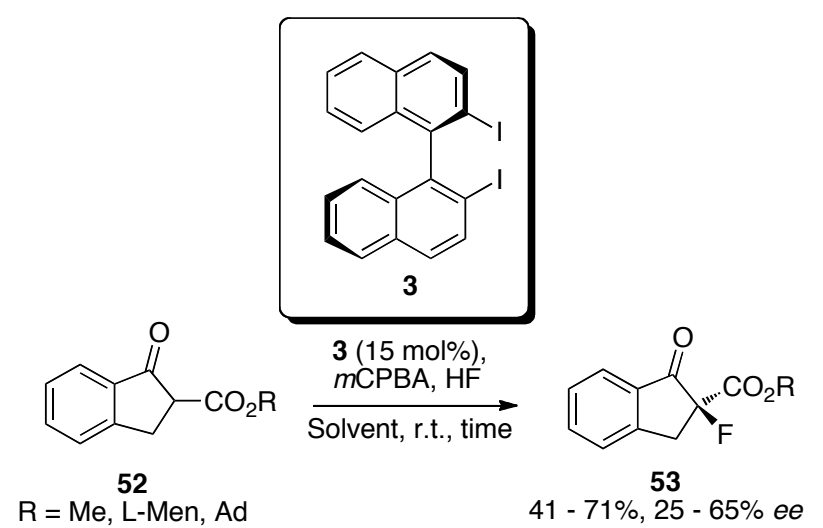

Scheme 22. Enantioselective $\alpha$-fluorination of ketoesters (1).

The mentioned transformation was significantly improved by Rueping. HF. $5 \mathrm{NEt}_{3}$ was identified as the most suitable fluorine source, and the lactate/resorcinol based iodine catalyst $9 \mathbf{b}$ was effectively modulated to its best variant introducing a D-menthyl ester group. The kinetic study of the reaction revealed that the ee of the product is invariable over the course of the fluorination indicating that no racemization is involved. The yields remained moderate for this fluorination, but the enantioselectivities were significantly improved, giving up to $92 \%$ ee for the fluorinated species 53 (Scheme 23). ${ }^{[38]}$ An interesting feature of the work consisted in the rationalization of the observed enantioselection by computational chemistry. Within reaction of the enolized form of the substrate, the calculations show that an I-O bonded intermediate $\mathbf{J}$ can be formed via $\mathbf{G}-\mathbf{I}$. After ruling out a direct nucleophilic attack of the fluoride to the $\alpha$ carbon due to the observed opposite stereochemistry of the products 53, the authors proposed a transition state $\mathbf{K}$, in which the iodine transfers from the carboxylic oxygen to the $\alpha$-carbon. Based on the calculations, transition state $\mathbf{J}$ is enantioselectivity-determining as the resulting intermediate $\mathbf{K}$ displays only a very low energy barrier. Also, the pro- $(R)$ TS is favored over its analogous pro- $(S) \mathrm{TS}$, according to the calculations. From there, a double inversion of stereochemistry takes place, in which Ar*-I is eliminated and a hemiacetale formed, which implies a first inversion of the configuration. trans-Selective opening by nucleophilic fluoride leads to the experimentally observed $(R)$-enantiomer of the product.
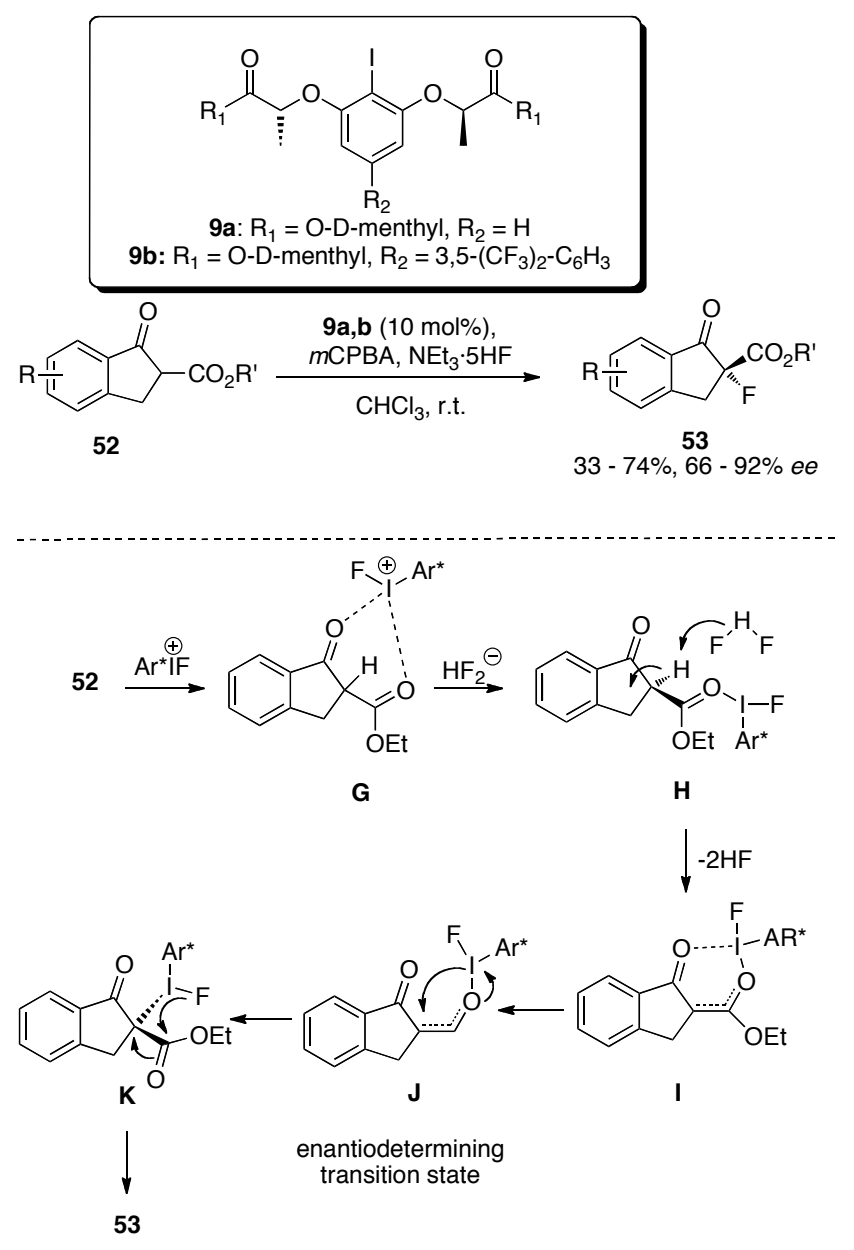

Scheme 23. Enantioselective $\alpha$-fluorination of ketoesters (2).

Investigation on additional $\alpha$-oxidation reactions of carbonyls have resulted in interesting enantioselective spiro-cyclization reactions. In 2014, Gong and coworkers successfully developed the first intramolecular asymmetric oxidative $\mathrm{C}\left(\mathrm{sp}^{2}\right)-\mathrm{C}\left(\mathrm{sp}^{3}\right)$ cross-coupling reaction of $N^{1}, N^{3}$ diphenylmalonamides $\mathbf{5 4}$, which is catalyzed by chiral iodide compounds. This stereoselective methodology features the consecutive functionalization of four $\mathrm{C}-\mathrm{H}$ bonds to access optically active spirooxindoles (Scheme 24). ${ }^{[52]}$ The hypervalent iodine 15a was generated in situ from the chiral aryliodine reagent 11b and peracetic acid. The attached tertiary amide moiety appeared to play a key role in the stereochemical induction. Moreover, as discussed in the section on supramolecular structure (vide supra), the chiral stereogenic lactic center had a major impact on the helical assembly formation and therefore on the stereochemistry of the product than the proline chirality. Based on previous studies, ${ }^{[53]}$ the authors suggested the formation of the stereogenic center by an 
intramolecular Friedel-Crafts-type addition followed by the oxidative cyclization via syn additionelimination favored through the $\mathrm{Si}$ face, giving product 55 with high enantioselectivity. Using density functional computation, Hadad, Sunoj and co-workers studied the detailed course of the stereoinduction with the resorcinol-based chiral iodine catalyst $\mathbf{1 1 b}$ used by Gong. ${ }^{[20]}$ The generation of a chiral environment around the iodine center, due to the presence of noncovalent interactions between the trifluoroacetate ligands and the amide moieties was already outlined above. From a mechanistic point of view, the substrate underwent two sequential catalytic cyclization steps. Hence, the chiral iodine participates twice in the cycle demonstrating a highly efficient role as a catalyst, particularly in the final enantioselectivity-determining cyclization (Scheme 24).

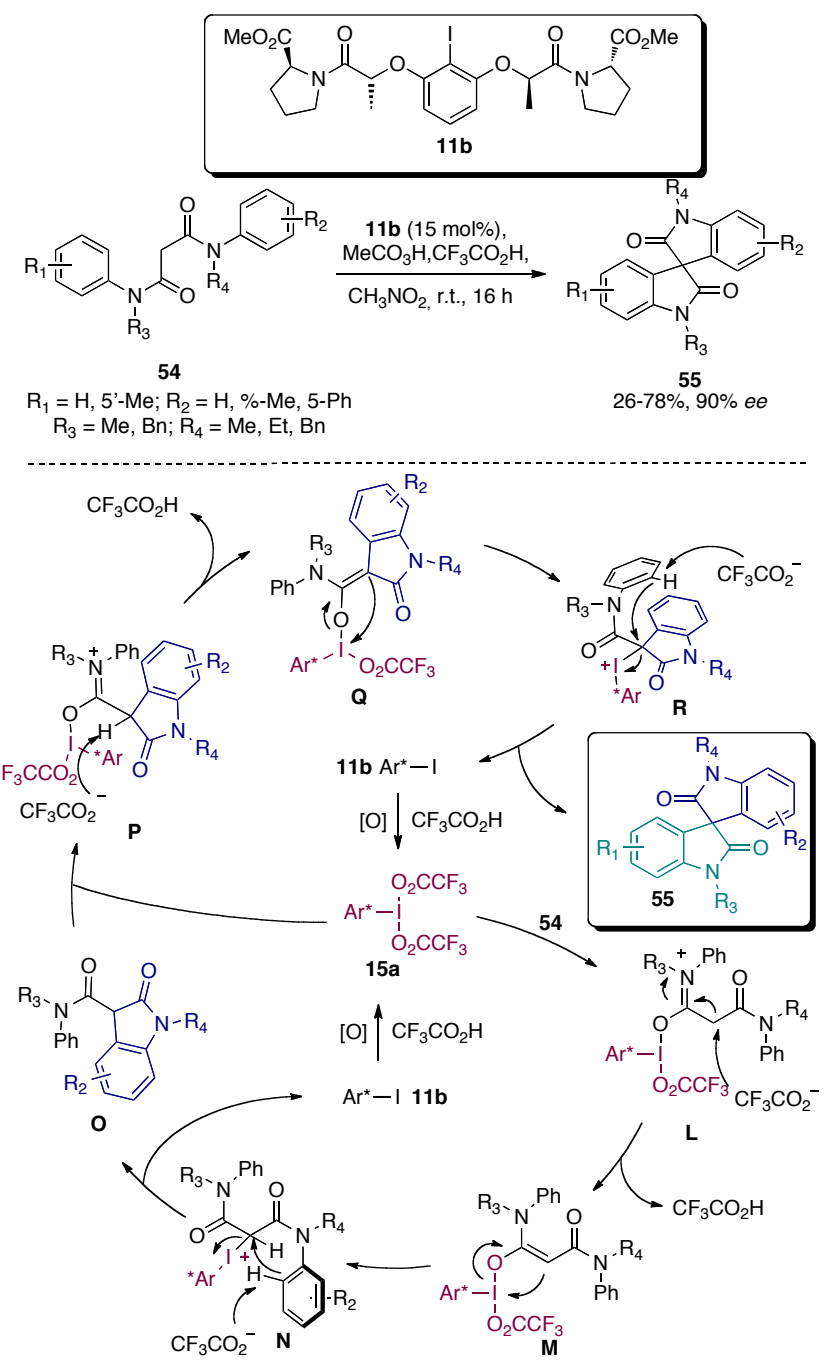

Scheme 24. Enantioselective $\mathrm{C}\left(\mathrm{sp}^{2}\right)-\mathrm{C}\left(\mathrm{sp}^{3}\right)$ cross-coupling reaction to form optically active spirooxindoles.

After the generation of the I(III) catalyst 15a, the catalytic cycle was suggested to start with the formation of $\mathbf{L}$ and an abstraction of a methylene proton. This promoted the formation of the Oiodonium intermediate $\mathbf{M}$, which went through an essential 1,3-migration to generate the low energy Ciodonium $\mathbf{N}$. This atypical reaction was considered the enantiocontrolling step. Next, an $\mathrm{S}_{\mathrm{N}} 2$ reaction between the ortho carbon of the $N 8$ '-phenyl group and the methylenic carbon took place forming the $\mathrm{C}-\mathrm{C}$ bond of the monooxindole structure $\mathbf{O}$. A similar mechanistic route via $\mathbf{P}-\mathbf{R}$ ended the second ring formation affording the desired chiral spiro-bisoxindole product 55. Due to the loss of chiral information arising from the second enolization to $\mathbf{Q}$ the overall stereoselectivity of the final product is solely dependent on the second ring closure sequence.

\subsection{Difunctionalization of Alkenes}

Due to the coordination ability of their electron-rich $\pi$-system, alkenes are suitable substrates for enantioselective oxidation with electrophilic chiral iodine catalysts. ${ }^{[1 \mathrm{e}, \mathrm{g}]}$ Alkenes represent a widespread functional group and their functionalization within the vicinal difunctionalization strategy is step-economic. Iodine(I/III) catalysis adds the aspect of an environmentally friendly synthesis. As a result of these attractive aspects, iodine catalyzed asymmetric difunctionalization reactions including oxygenations, aminations and fluorinations have started to appear more and more often throughout the last decade.

Taking into account that alkenes can also react with the stoichiometric oxidant destined for the iodine oxidation, the basic requirement for chemo- and/or enantioselectivity in these I(I)/I(III) systems is either a chemoselective oxidation of the aryliodine(I) catalyst or a significant kinetic preference for the catalyzed pathway over the non-catalyzed background reaction.

In 2012, Fujita developed the first example of an asymmetric difunctionalization of internal alkenes $\mathbf{5 7}$ employing a chiral iodoarene $9 \mathrm{c}$ in a $10 \mathrm{~mol} \%$ catalyst loading and a peracid as the co-oxidant. In this case, the starting material contained both oxygen atoms that were oxidatively transferred to the alkene moiety. With the generated lactate-based resorcinol catalyst 56, they employed this strategy in the first stereoselective total synthesis of hydroxymonocerins $\mathbf{6 0 , 6 1}$ from preformed alkenylbenzoate substrates 57 . The reaction went through a cascade formation of the oxolane-fused isochroman-1-one backbone by a double oxygenative cyclization. The presence of the iodine(III) catalyst was essential for the asymmetric intramolecular oxylactonization, as the stereochemical outcome relied on the prochiral face recognition through catalyst control. ${ }^{[16 \mathrm{~d}]}$ Therefore, the preinstalled stereogenic centers of the substrate do not dominate the stereochemistry of the cyclisation event (Scheme 25). ${ }^{[16 c]}$ The exact courses of for enantioselective induction in these reactions were discussed recently by Fujita. ${ }^{[54]}$ 


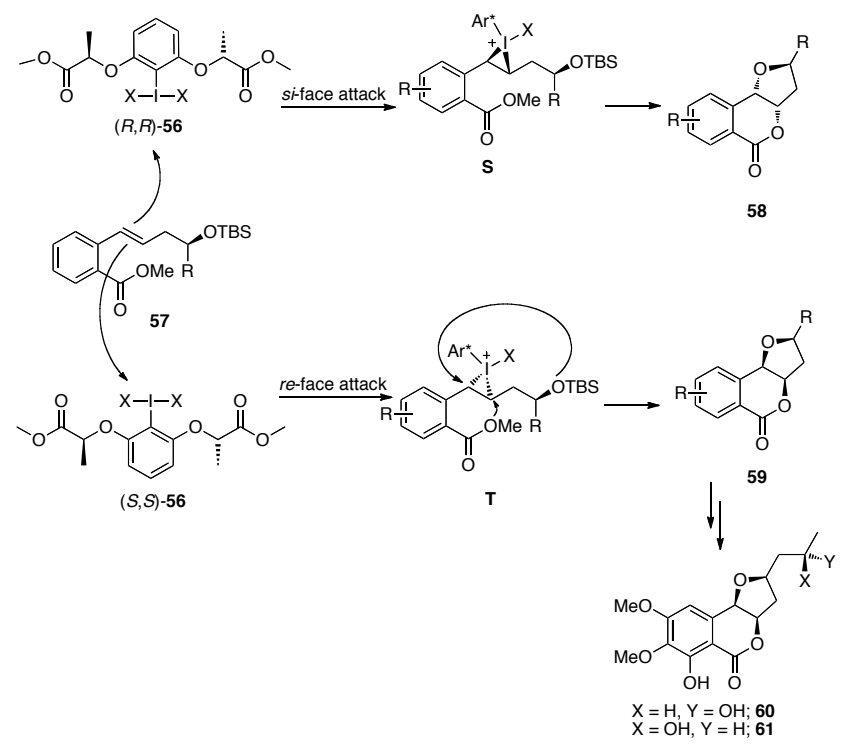

Scheme 25. Stereoselective total synthesis of hydroxymonocerins.

Their ensuing study was based on the application of the developed asymmetric methodology to synthesize optically active 4-hydroxyisochroman-1-ones 63,64. In this case, four possible stereoisomers could be achieved in different ratios depending on the substrate and the chiral hypervalent iodine, for which 4 was chosen. Syn-selectivity was preferred by I(III)catalyzed reactions giving products 63 and 64 with significant enantiomeric excess through a Woodwardtype mechanism. In contrast, the background antidioxygenation under the assistance of $m \mathrm{CPBA}$ originated from a Prévost mechanism producing racemic diastereomers 65 and 66 (Scheme 26). ${ }^{[16 \mathrm{e}]}$

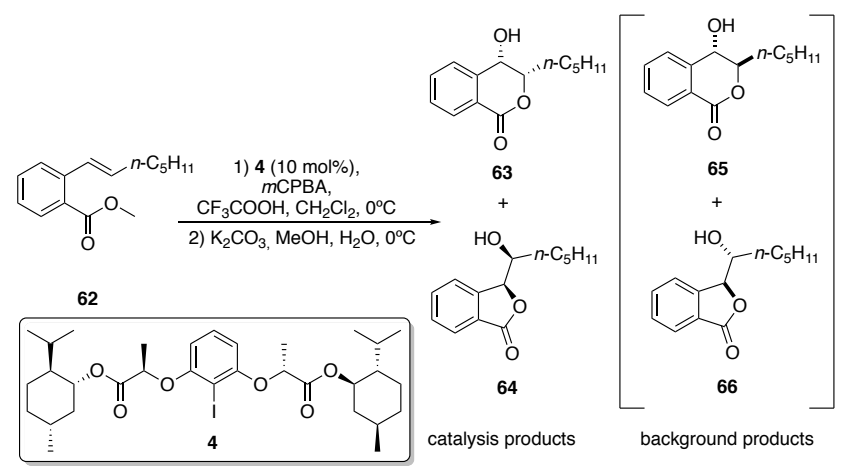

Scheme 26. Enantioselective synthesis of optically active 4hydroxyisochroman-1-ones.

The same concept can be applied to the formation of useful chiral heterocycles, such as oxazolines and dihydrooxazines within intramolecular cyclization onto prochiral faces of an alkene. In 2015, Moran reported an oxidative cyclization of $N$-alkenylamides 67 using $10 \mathrm{~mol} \%$ of a chiral iodine pre-catalyst 11c and 2 equivalents of Selectfluor as the terminal oxidant. Through this route, five and six-membered rings were obtained, corresponding to oxazolines and dihydrooxazines. Yields and enantioselectivities ranged from moderate to good. ${ }^{[5]}$ The mechanism involved the in situ generation of iodine(III) catalyst, which activated the alkene for an intramolecular attack by the amide oxygen. The subsequent intermolecular nucleophilic oxygenation at the carbon-iodine bond of $\mathbf{U}$ regenerates the pre-catalyst 11c and releases the cyclized product 68 (Scheme 27).

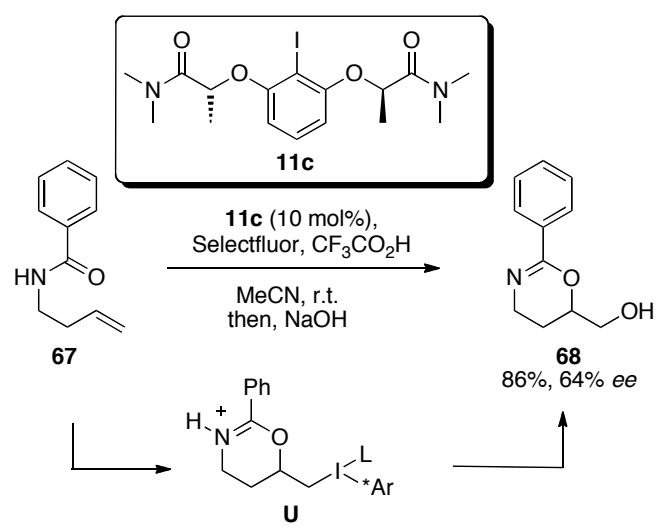

Scheme 27. Enantioselective oxidative cyclization of $N$ alkenylamides.

Masson investigated the first enantioselective sulfonyl- and phosphoryl-derived oxylactonization of non-rigid 4-pentenoic acid derivatives. By using 20 $\mathrm{mol} \%$ of a chiral iodine precursor $\mathbf{1 0 a}, m \mathrm{CPBA}$ as cooxidant and sulfonic or phosphoric acid as nucleophiles, the desired products $\mathbf{7 0}$ and 71 were readily afforded. The common $C_{2}$-symmetric resorcinol iodine compound $\mathbf{1 0 a}$ with a secondary lactamide moiety including a bulky 2,4,6-trimethyl phenyl group provided good yield and enantioselectivity in both cases (Scheme 28). ${ }^{[56]}$

From a mechanistic point of view, the active aryl- $\lambda^{3}-$ iodane species of the Koser type underwent an electrophilic addition to the double bond, forming a chiral iodonium intermediate V. After the lactonisation, nucleophile exchange at iodine of $\mathbf{W}$ and subsequent nucleophilic addition within $\mathbf{X}$ by a sulfonyl or phosphoryl group, the final $\gamma$-lactones $\mathbf{7 0}$ and 71 were formed. In the determining transition state of $\mathbf{V}$, the blockage of the $S i$ face of the substrate by the catalyst is accomplished through $n-\sigma^{*}$ interactions between the iodine(III) center and the carbonyl oxygen of the amide. This effective selection provides the observed absolute enantioselective outcome (Scheme 28). 


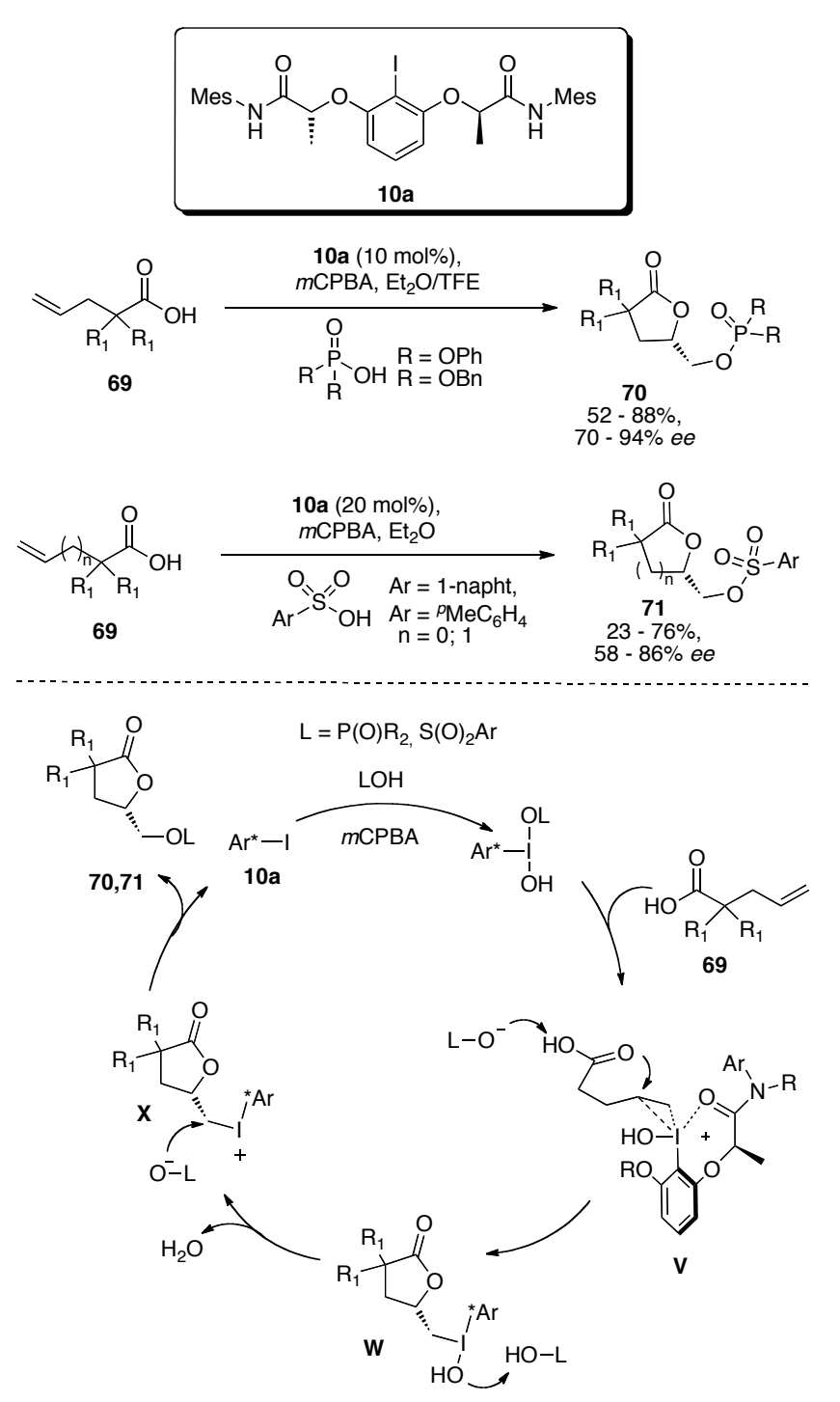

Scheme 28. Enantioselective oxidative cyclization of $\omega$ alkenylcarboxylic acids.

After successfully introduction of the stoichiometric application of different chiral iodine(III) reagents, ${ }^{[57]}$ in 2014 Wirth described the first catalytic intramolecular diamination of alkenes 72. By using chiral hypervalent iodine catalysis with $\mathbf{4}$, which included an oxidative cyclization step followed by a reduction, they obtained the corresponding diamines $\mathbf{7 3}$ with up to $86 \%$ ee (Scheme 29). ${ }^{[58]}$ Although a detailed structural elucidation of the active iodine(III) catalyst remains a future task, one can anticipate a rigid stereochemical environment arriving from coordination of the pyridine to the iodine(III) center. ${ }^{[9 j]}$ Related coordination was recently confirmed in an achiral iodine(I/III) catalyzed diacetoxylation and matches the concept of intramolecular coordination at iodine(III). ${ }^{[99]}$

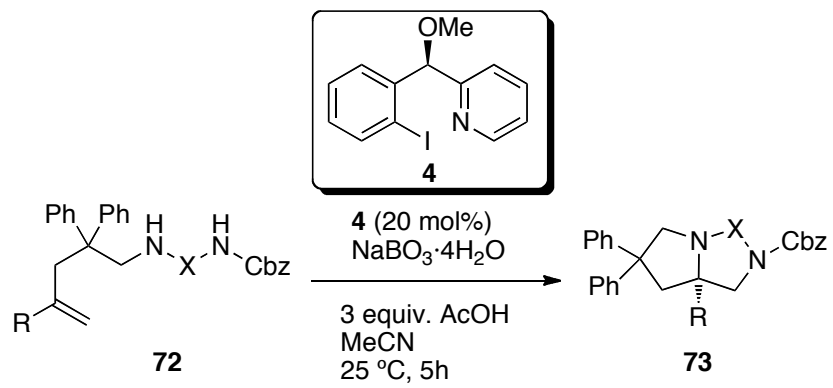

Scheme 29. Catalytic intramolecular diamination of alkenes.

One of the first stoichiometric examples of alkene fluorination by the action of hypervalent iodine was reported in 2012 in the form of an intramolecular aminofluorination, in which the fluorination proceeds in the presence of HF.Py as fluorine source and $\mathrm{BF}_{3} \cdot \mathrm{OEt}_{2}$ as an activator. ${ }^{[60]}$ Based on this work, Nevado developed the enantioselective transformation using a chiral hypervalent difluoroiodine(III) reagent, obtaining the desired fluorinated piperidine derivatives with up to $88 \% e e .^{[61]}$ Subsequently, Kita and Shibata developed a catalytic asymmetric version of the mentioned reaction, in which axially chiral bisiodine(III) is the in situ generated from $\mathbf{3}$ under the combined action of $m$ CPBA and HF. Nevertheless, the yields were relatively lower than the ones from the previous stoichiometric transformation (43-65\% yield) and the same concerned the enantioselectivities of products $75(0-70 \%$ ee $)$ (Scheme 30$){ }^{[51]}$

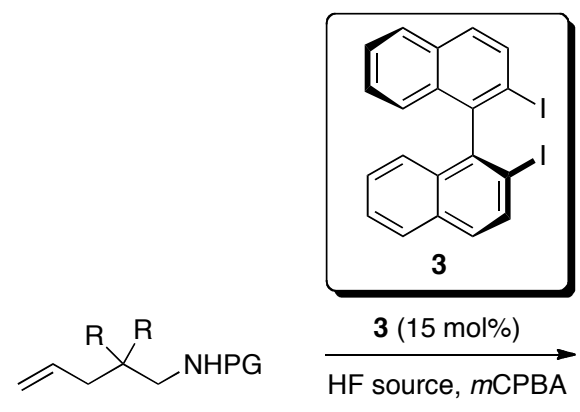

74

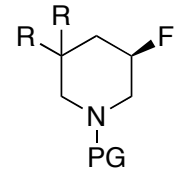

75
(3 examples)

Scheme 30. Catalytic enantioselective version of the aminofluorination reaction.

The most recent development in the intramolecular aminofluorination of arylated allylic amines was introduced by Jacobsen. ${ }^{[62]}$ The use of chiral iodine(III) catalysis with 9d provided elegant access to $\alpha$ fluoroaziridines 77 with up to $97 \%$ ee. Subsequent aziridine ring opening of these building blocks with a range of nucleophiles yielded the analogous linear structures with vicinal fluoroamino group in excellent yields and enantioselectivities. Although 4- and 6membered rings could not be formed, the methodology 
was successfully extended to one example of a 5membered ring 79. The syn/anti difference regarding the relative stereochemistry of the products $\mathbf{7 7}$ and $\mathbf{7 9}$ was postulated to be the consequence of two different pathways for the individual substrate classes, being both successfully promoted by the same catalyst 9d, which exercises high to complete face selection.

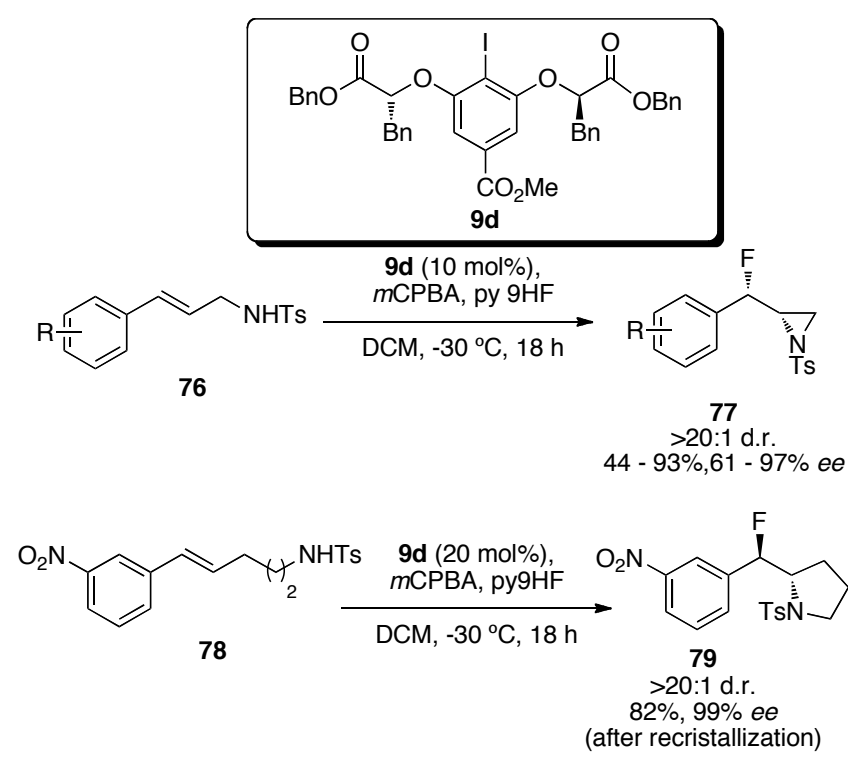

Scheme 31. Catalytic enantioselective fluoroamination reactions.

In the same context, Jacobsen elaborated an enantioselective formation of 4-fluoroisochroman-1one $\mathbf{8 1}$ by the action of a 2-iodoorcinol-based catalyst 9e. The reaction accesses lactones incorporating fluorinated stereogenic centers with high diastereoand enantioselectivity. Alkyl, halide, or trifluoromethoxy substitution pattern at the 6-, 5-, and 4-positions of $\mathbf{8 0}$ were generally well tolerated, with the fluorolactone products $\mathbf{8 1}$ obtained in $80-96 \%$ ee. However, substrates $\mathbf{8 0}$ carrying more electrondeficient trifluoromethyl or carbomethoxy groups led to reduced yields and enantioselectivities (Scheme 32). ${ }^{[63]}$

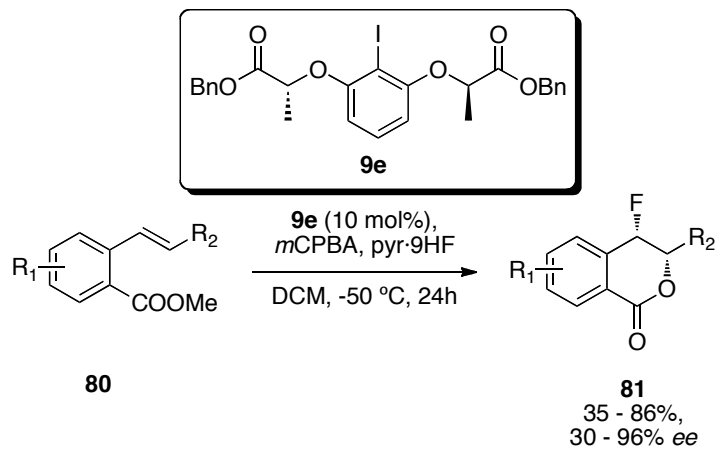

Scheme 32. Catalytic enantioselective fluorolactonization.
In 2016, Muñiz and Ishihara extended the use of non-racemic hypervalent iodine catalysis to the intermolecular diacetoxylation of alkenes and thereby demonstrated the feasibility of iodine catalysis in enantioselective alkene oxidation under full intermolecular reaction control. The work has precedence regarding a transformation that is stoichiometric in chiral iodine(III) reagent. However, the catalytic variant relied on structurally defined catalysts. Fujita $^{[16 c]}$ and Ishihara ${ }^{[23]}$ firstly suggested the spatial disposition of some hypervalent iodine(III) reagents of the resorcinol lactate, while the present work offered the first experimental rationalization and exploitation of the resulting supramolecular assembly and its application to intermolecular reaction control.

Based on the modular toolbox approach discussed above in Figure 3, the required reactivity of the iodine catalysts was fine-tuned for the mentioned diacetoxylation reactions.

The first methodology is based on the use of catalyst 10d in combination with peracetic acid as the terminal oxidant. This catalyst bearing a methyl group at the 4position of the aryl backbone and an $N$-arylamide moiety at the arms performed kinetically superior than related candidates. High yields and enantiomeric excesses were provided when terminal styrene derivatives 82 were employed as substrates (Scheme 33). As expected, the choice of peracetic acid as oxidant excluded potential contribution via an undesired background epoxidation. ${ }^{[19]}$ The presence of a catalytic amount of triflic acid was found beneficial. In agreement with the mechanistic insight by Gade, ${ }^{[64]}$ the presence of this Brønstedt acid is crucial for the kinetic dominance of the chirally catalyzed pathway. At the stage of the diacetoxy iodine(III) catalyst stage, $\mathrm{TfOH}$-promoted protonolysis removes one of the acetate ligands and thereby provides the free site for the selective coordination of one the prochiral faces of the substrate. This step includes partial loss of the helical arrangement from hydrogen bonding, but due to the remaining chiral pocket of $\mathbf{1 3 b}$, enantiodiscrimination remains excellent.

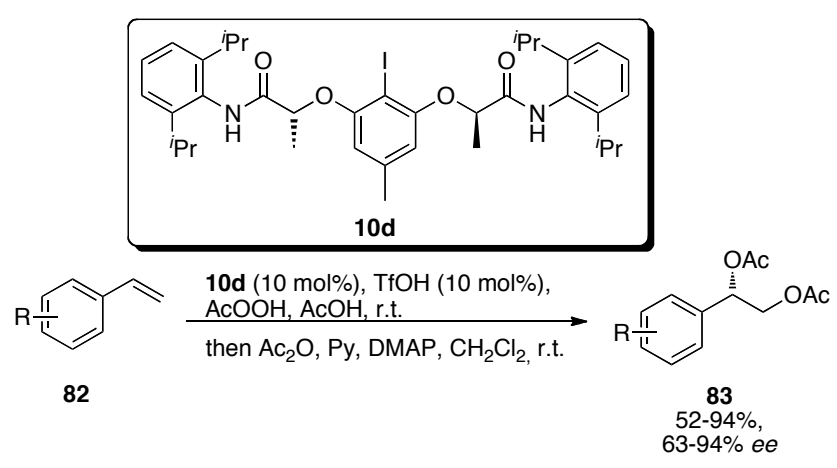

Scheme 33. Enantioselective diacetoxylation of terminal styrene derivatives. 


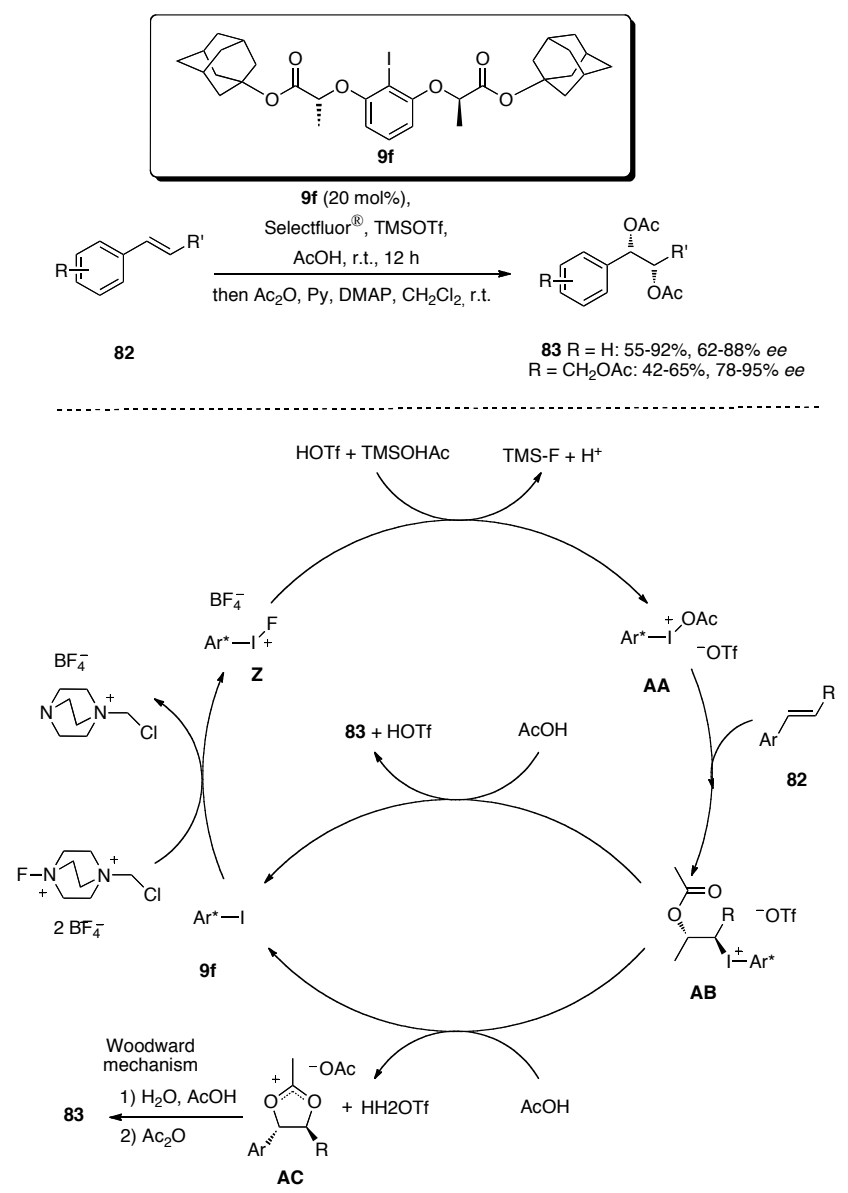

Scheme 34. Enantioselective diacetoxylation of terminal and internal styrene derivatives.

As for the enantioselective intermolecular diacetoxylation with peracetic acid, Selectfluor ${ }^{\mathbb{B}}$ can also be employed as the terminal oxidant. In this case, the active iodine(III) catalyst is generated from precatalyst 9f containing a 1-adamantyl ester moiety in combination with TMSOTf, which exercises the same role as the HOTf in the previous reaction. This diacetoxylation variant was suitable for both terminal styrenes and internal derivatives from cinammyl alcohols and again afforded good enantioselectivities and yields (Scheme 34$){ }^{[65]}$

The catalytic cycle starts with the oxidation of the chiral iodoarene $9 \mathbf{f}$ and by the action of trimethylsilyl acetate and triflic acid generates the active cationic state of the chiral I(III) catalyst AA. Upon addition of the styrene substrate 82, AA exercises face recognition and the generated enantioenriched iodooxygenated intermediate $\mathbf{A B}$ from acetate incorporation is identical to the one from catalytic diacetoxylation of styrenes with peracetic acid. It provides the final product within two pathways: a Woordward mechanism through the formation of a dioxolonium derivative $\mathbf{A C}$ arising from intramolecular displacement of the iodine(III), or by a direct reductive deiodination through an intermolecular $\mathrm{S}_{\mathrm{N}} 2$ reaction using a second acetate nucleophile. As determined by control experiments, both pathways are operative, although the intramolecular scenario usually outperforms the intermolecular one.

Gilmour reported in 2016 the enantioselective vicinal difluorination of alkenes $\mathbf{8 4 , 8 6}$ using a chiral, non-racemic aryliodide derivative 11e for face differentiation of prochiral allylic ethers. ${ }^{[66]}$ Subsequent work revealed the important insight that lactate-derived catalysts in combination with Selectfluor can be employed in order to exercise effective intermolecular reaction control and obtain fluorinated species such as $\mathbf{8 5}$ and $\mathbf{8 7}$ with enantiomeric excess (Scheme 35). ${ }^{[67]}$

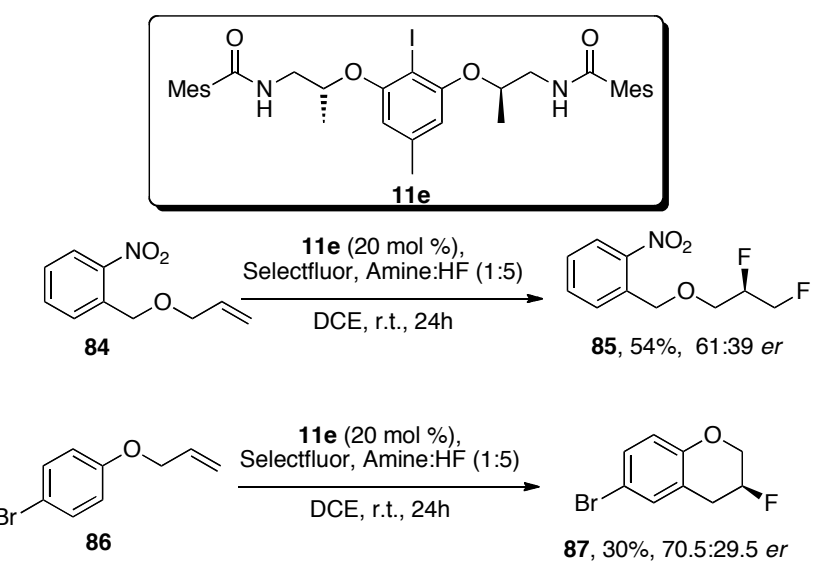

Scheme 35. Enantioselective fluorination.

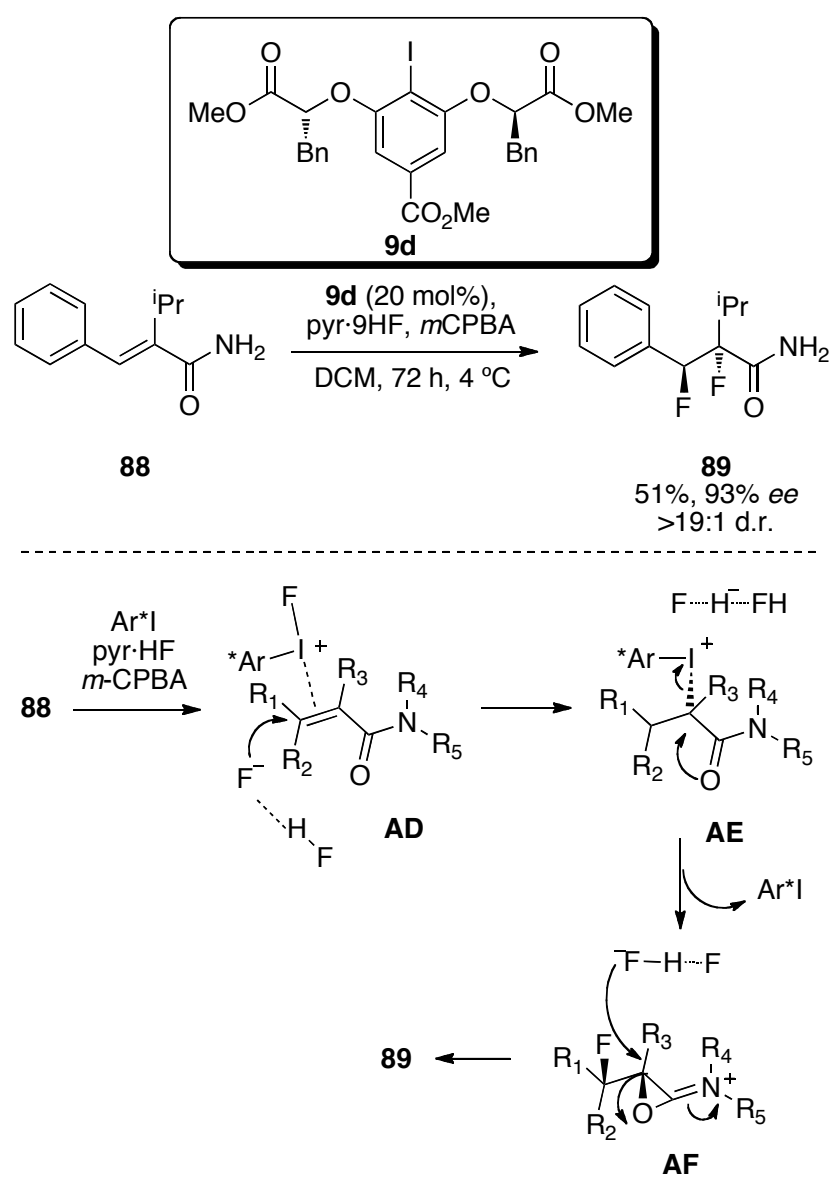


Scheme 36. Enantioselective difluorination of acrylamides.

Jacobsen disclosed similar reactivity for substrate $\mathbf{8 8}$. The method uses a nucleophilic fluorine source and the conventional oxidant $m \mathrm{CPBA}$ in conjunction with the related chiral iodine(I) catalyst 9d (Scheme 36). The proposed catalytic cycle consists in the formation of the chiral iodine(III) catalyst, which oxidizes the alkene in a stereoselective way via AD. Highly electron-deficient $\beta$-monosubstituted styrenes yielded the corresponding $s y n$-difluorination products, while $o$-nitrostyrene derivatives and $\beta, \beta$-disubstituted acrylamides $\mathbf{8 8}$ gave anti-difluorination, which is believed to arise from anchimeric assistance by the vicinal polar groups. Moreover, the difluorination of trisubstituted cinnamate derivatives provided a wide range of the desired products with 19:1 d.r. and 97\% $e e$, respectively. The use of arenes and amides with alternative substitution gave different stereochemical outcomes in the products. ${ }^{[68 \mathrm{a}]}$ Another example of an unprecedented alkene oxidation was developed by Jacobsen, who reported a unique catalytic approach to geminal difluorination products 91 (Scheme 37). ${ }^{\text {68b] }}$ This transformation involves an initial iodofluorination of 90 to difunctionalized AG. The required reductive elimination of the aryliodine(III) does not arrive from the common nucleophilic addition, but is trigged by an aryl migration involving the phenonium ion $\mathbf{A H} .^{[0 \mathrm{k}]}$ This concept was previously introduced by Wirth in the oxidation reaction of chalcones under stoichiometric iodine(III) control. ${ }^{[69]}$ Upon fluoride addition, the subsequent rearrangement forms the final gemdifluoromethylated product 91 in high yields and enantioselectivities. Again, catalyst fine-tuning is accomplished within the resorcinol/lactate combination and the introduction of benzylic moieties in the chiral position of the catalyst 9d was crucial for high reactivity, and ultimately the enantioselectivity, due to the decrease in reaction temperature. In addition to the present catalytic transformation, enantioselective transformations stoichiometric in iodine(III) have been elaborated by Wirth. ${ }^{[69,70]}$ In addition, an investigation on the electronic effect of a $4-\mathrm{CF}_{3}$ substituent at the central aryl group of the idoine(III) reagent was provided recently. ${ }^{[71]}$

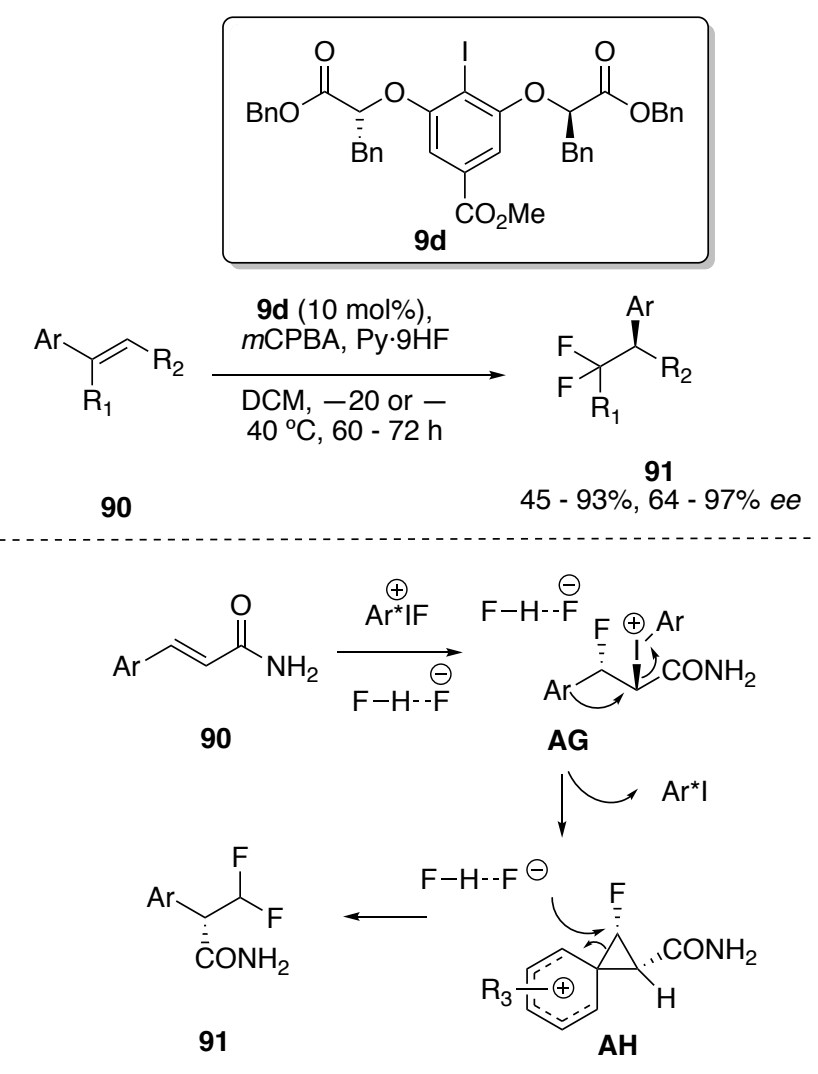

Scheme 37. Enantioselective geminal difluorination.

Building on a body of achiral reactions, ${ }^{[35 a, 72]}$ Muñiz had already developed in 2011 an enantioselective stoichiometric diamination of styrene and derivatives 82 using chiral iodine(III) reagents. Up to $95 \%$ ee could be obtained under these conditions. ${ }^{[72 b]}$ This high enantiomeric induction rose the question for catalytic reaction conditions.

Recurring to the lactate/resorcinol toolbox for catalyst optimization, the 4-methyl aryl iodide 11e incorporating two chiral lactamide side chains resulted as the optimum catalyst precursor. Suitable conditions for the desired iodine(I/III) redox catalysis employed 3-chloroperbenzoic acid ( $m$ CPBA) as terminal oxidant, which in the optimized MTBE/HFIP solvent combination showed only negligible background epoxidation. In this way, reaction proceeded in excellent enantioselectivity with up to $98 \%$ ee for terminal and internal styrenes (Scheme 38). ${ }^{[15 c]}$ This protocol pioneers enantioselective catalytic diamination of alkenes under intermolecular reaction control. As demonstrated for a vinyl steroid, the reaction proceeds under complete catalyst control for chiral substrates. The resulting vicinal diamines can be readily transformed into the corresponding free 1-aryl ethylene diamines, which represent useful building block in synthesis. This context had been demonstrated for the synthesis of alkaloid levamisole. ${ }^{[73]}$ 


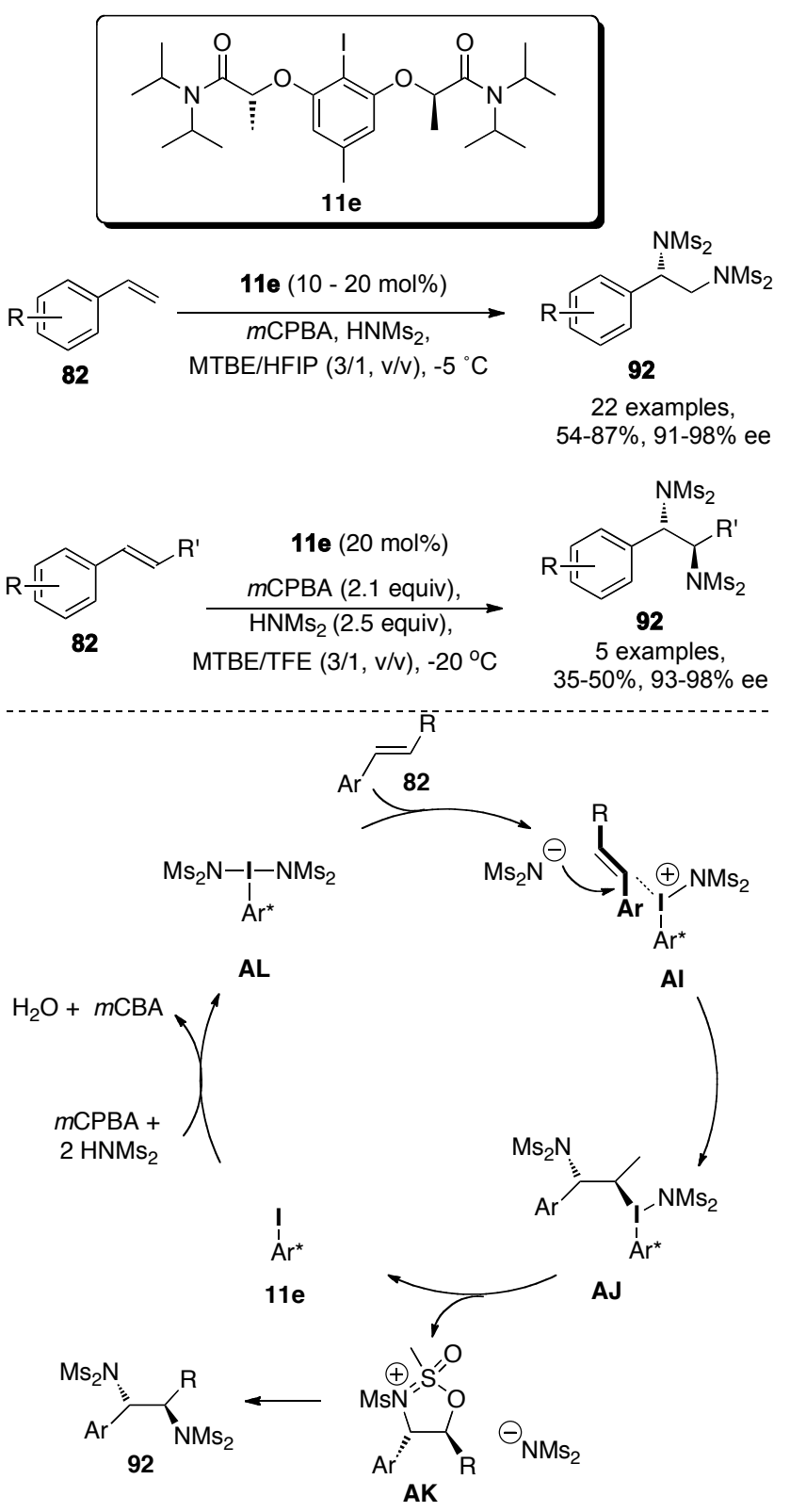

Scheme 38. Enantioselective vicinal diamination.

Mechanistically, the diamination should proceed through an iodine(III) catalyst state $\mathbf{A L}$ with two incorporated bismesylimides at iodine. Here, the enhanced dissociation capacity of the bismesylimide is decisive, as it readily promotes a free coordination site at the iodine center for efficient enantiotopic face differentiation within substrate coordination AI. The obtained high enantioselectivity originates from direct amination at the benzylic position followed by intramolecular de-iodination at stage $\mathbf{A J}$ to dioxooxathiazolidinium AK, which upon addition of the remaining bismesylimide provides the final products 92 as single diastereoisomers in excellent ee. It is interesting to compare the stereochemical course of the diacetoxylation and diamination reactions. For a given absolute configuration of the lactic side chain of the catalyst, both catalysts form identical supramolecular helical environments and thus provide the same face selection of the styrene substrate providing identical absolute benzylic $(S)$-configuration of the products. The major difference rests with the chiral pockets within the helical assembly of the catalysts. Inspection of the crystal structures from Figure 2 reveals a significantly wider pocket for the bisamide 14a, which adopts to the required spatial trajectory for the bissulfonimide nucleophile, which exercise a larger steric requirement than the acetate nucleophile in the cases of catalysts 13a and 13b (Scheme 39).
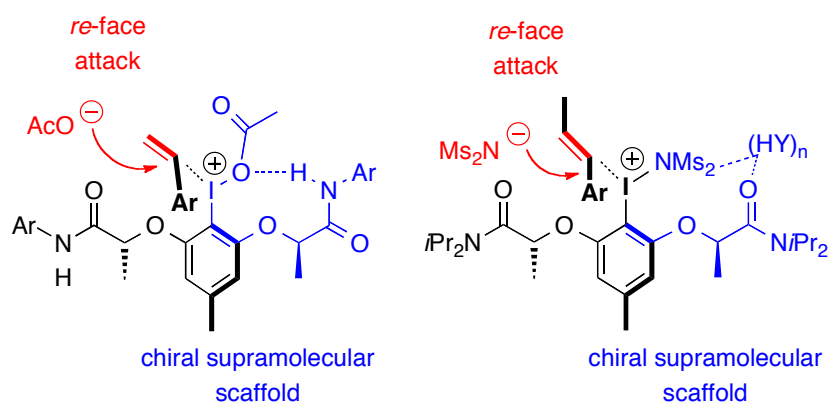

Scheme 39. Efficient face selection within individual chiral supramolecular scaffols of catalysts related to $\mathbf{1 3 a} \mathbf{a}, \mathbf{b}$ and $14 a$.

These results allow for a first prediction of absolute chirality of products within a given catalytic oxidation by chiral aryliodine(III) catalysts. They hold strong promise for a possible future rationale design of catalysts deriving from the lactate/resorcinol motif. Clearly, more data on catalyst structure and reaction transition states are required. Yet at the present stage, this particular catalyst concept holds great promise as evidenced from the large number of successful applications discussed in the previous sections.

\section{Conclusion}

The status quo of iodine(I/III) oxidation catalysis has reached a high level. It has proven to be applicable to enantioselective reactions under intra- and intermolecular reactions control tolerating a wide range of different transferable groups including oxygen atoms from alcohols and different kinds of aids, nitrogen atoms from amides and fluorine atoms. The quest for effective enantioselective oxidation has resulted in a large number of chiral iodine catalysts, which have been optimized both with respect to structure and cooperation with the respective terminal oxidant. Extensive efforts have been undertaken to arrive at an advanced structure-reactivity relationship for several of the chiral iodine catalysts, which in some cases has already permitted the design of individual iodine catalysts for specific transformations. Continuous catalyst structure optimization within the lactate/resorcinol family as well as the identification of 
new catalyst structures will certainly continue within the near future. The application of specifically designed chiral aryliodine(I/III) catalysis has emerged only recently, but already at the present stage holds very high promise to become a rather general tool in enantioselective oxidation catalysis.

\section{Acknowledgements}

The authors thank all current and past co-workers of the Muñiz group for their experimental and intellectual contributions to this research project. Their names are provided in the references. The authors are grateful to the Spanish Ministerio de Economia y Competitividad for continuous support of their research.

\section{References}

[1] a) R. Noyori, Asymmetric Catalysis In Organic Synthesis, John Wiley \& Sons, Chichester, 1994; b) Catalytic Asymmetric Synthesis (Ed.: I. Ojima), WileyVCH, Weinheim 2000; c) Transition Metals for Organic Synthesis (Eds.: M. Beller, C. Bolm), Wiley-VCH, Weinheim 2004; d) Comprehensive Asymmetric Catalysis (Eds: E. N. Jacobsen, A. Pfaltz, H. Yamamoto), Springer, Berlin 1999; e) Asymmetric Catalysis on Industrial Scale, (Ed.: H.-U. Blaser, E. Schmidt), Wiley-VCH, 2004.

[2] T. P. Yoon, E. N. Jacobsen, Science 2003, 299, 16911693.

[3] Q.-L. Zhou, Privileged Chiral Ligands and Catalysts, Wiley-VCH, Weinheim 2011.

[4] D. J. Berrisford, C. Bolm, K. B. Sharpless, Angew. Chem. 1995, 107, 1159 - 1171; Angew. Chem. Int. Ed. Engl. 1995, 34, 1059-1070.

[5]a) A. Messerschmidt, R. Huber, K. Wieghardt, T. Poulos, Handbook of Metalloproteins, Wiley, New York, 2001; b) Nature Insight Metalloproteins, Nature 2017, 460, 813-862.

[6] a) M. T. Reetz, Directed Evolution of Selective Enzymes: Catalysts for Organic Chemistry and Biotechnology, Wiley VCH, Weinheim, 2016; b) H. Renata, J. Wang, F. H. Arnold, Angew. Chem. 2015, 127, 3408-3426; Angew. Chem. Int. Ed. 2015, 54, 3351-3367; c) M. T. Reetz, J. Am. Chem. Soc. 2013, 135, 12480-12496; d) M. T. Reetz, Angew. Chem. 2011, 123, 144-182; Angew. Chem. Int. Ed. 2011, 50, 138-174.

[7] H. Gröger, A. Berkessel, Asymmetric organocatalysis - from biomimetic concepts to applications in asymmetric synthesis, Wiley-VCH, Weinheim, 2005.

[8] a) O. A. Wong, Y. Shi, Chem. Rev. 2008, 108, 39583987; b) Y. Zhu, Q. Wang, R. G. Cornwall, Y. Shi, Chem. Rev. 2014, 114, 8199-8256.

[9] a) V. V. Zhdankin, Hypervalent Iodine Chemistry: Preparation, Structure, and Synthetic Applications of Polyvalent Iodine Compounds; Wiley, Chichester, UK,
2014; b) Hypervalent Iodine Chemistry. (Ed.: T. Wirth), Top. Curr. Chem. 373, Springer, Berlin, 2016; c) A. Yoshimura, V. V. Zhdankin, Chem. Rev. 2016, 116, 3328-3435; d) Hypervalent Iodine Chemistry. Modern Developments in Organic Synthesis (Ed.: T. Wirth), Top. Curr. Chem. 224, Springer, Berlin, 2003; e) V. V. Zhdankin, P. J. Stang, Chem. Rev. 2002, 102, 2523-2584; f) V. V. Zhdankin, P. J. Stang, Chem. Rev. 2008, 108, 5299-5358; g) R. M. Moriarty, O. Prakash in Organic Reactions, John Wiley \& Sons, Inc.: Hoboken, USA, 2001; pp 327-415; h) A. Varvoglis, Hypervalent iodine in organic synthesis, (Eds.: O. Meth-Cohn, A. Katritzky, C. Rees), Academic Press, 1997; i) T. Wirth, Angew. Chem. 2005, 117, 3722-3731; Angew. Chem. Int. Ed. 2005, 44, 3656-3665.; j) R. D. Richardson, M. Desaize, T. Wirth, Chem. Eur. J. 2007, 13, 6745-6754.; k) M. Uyanik, K. Ishihara, Chem. Commun. 2009, 0, 20862099; 1) A. Yoshimura, M. S. Yusubov, V. V. Zhdankin, Org. Biomol. Chem. 2016, 14, 4771-4781; m) F. Singh, T. Wirth, Synthesis 2013, 45, 2499-2511.

[10] a) M. Ochiai, Chem. Rec. 2007, 7, 12-23; b) M. Ochiai, K. Miyamoto, Eur. J. Org. Chem. 2008, 2008, 42294239; c) T. Dohi, Y. Kita, Chem. Commun. 2009, 2073-2085.

[11] a) F. Berthiol, Synthesis 2015, 47, 587-603; b) R. Kumar, T. Wirth, Top. Curr. Chem. 2015, 373, 243261; c) M. Ngatimin, D. W. Lupton, Aust. J. Chem. 2010, 63, 653-658; d) R. M. Romero, T. H. Wöste, K. Muñiz, Chem. Asian J. 2014, 9, 972-983; e) M. Uyanik, K. Ishihara, J. Synth. Org. Chem. Japan 2012, 70, 1116-1122; f) F. V. Singh, T. Wirth, Chem. Asian J. 2014, 9, 950-971.

[12] a) Y. Yamamoto, Y. Kawano, P. H. Toy, H. Togo, Tetrahedron 2007, 63, 4680-4687; b) S. M. Altermann, R. D. Richardson, T. K. Page, R. K. Schmidt, E. Holland, U. Mohammed, S. M. Paradine, A. N. French, C. Richter, A. M. Bahar, Eur. J. Org. Chem. 2008, 5315-5328; c) W.-T. Wu, L. Zhang, S.-L. You, Chem. Soc. Rev. 2016, 45, 1570-1580.

[13] a) S. Quideau, G. Lyvinec, M. Marguerit, K. Bathany, A. Ozanne-Beaudenon, T. Buffeteau, D. Cavagnat, A. Chénedé, Angew. Chem. 2009, 121, 4675-4679; Angew. Chem. Int. Ed. 2009, 48, 4605-4609; b) S. Brenet, F. Berthiol, J. Einhorn, Eur. J. Org. Chem. 2013, 8094-8096; c) T. Dohi, H. Sasa, K. Miyazaki, M. Fujitake, N. Takenaga, Y. Kita, J. Org. Chem. 2017, 82, 11954-11960.

[14] a) K. A. Volp, A. M. Harned, Chem. Commun. 2013, 49, 3001-3003; b) S. J. Murray, H. Ibrahim, Chem. Commun. 2015, 51, 2376-2379; c) A. Rodríguez, W. J. Moran, Synthesis 2012, 44, 1178-1182.

[15] a) R. D. Richardson, T. K. Page, S. Altermann, S. M. Paradine, A. N. French, T. Wirth, Synlett 2007, 4, 538542; b) M. Uyanik, T. Yasui, K. Ishihara, Angew. Chem. 2010, 122, 2221-2223; Angew. Chem. Int. Ed. 2010, 49, 2175-2177; c) K. Muñiz, L. Barreiro, R. M. 
Romero, C. Martínez, J. Am. Chem. Soc. 2017, 139, 4354-4357.

[16] M. Fujita, S. Okuno, H. J. Lee, T. Sugimura, T. Okuyama, Tetrahedron Lett. 2007, 48, 8691-8694; b) M. Fujita, Y. Ookubo, T. Sugimura, Tetrahedron Lett. 2009, 50, 1298-1300; c) M. Fujita, Y. Yoshida, K. Miyata, A. Wakisaka, T. Sugimura, Angew. Chem. 2010, 122, 7222-7225; Angew. Chem. Int. Ed. 2010, 49, 7068-7071; d) T. Takesue, M. Fujita, T. Sugimura, H. Akutsu, Org. Lett. 2014, 16, 4634-4637; e) M. Shimogaki, M. Fujita, T. Sugimura, Eur. J. Org. Chem. 2013, 7128-7138; f) M. Fujita, K. Mori, M. Shimogaki, T. Sugimura, RSC Adv. 2013, 3, 17717-17725; g) M. Fujita, K. Mori, M. Shimogaki, T. Sugimura, Org. Lett. 2012, 14, 1294-1297; h) M. Fujita, M. Wakita, T. Sugimura, Chem. Commun. 2011, 47, 3983-3985.

[17] M. Uyanik, T. Yasui, K. Ishihara, Tetrahedron 2010, $66,5841-5851$.

[18] M. Uyanik, T. Yasui, K. Ishihara, Angew. Chem. 2013, 125, 9385-9388; Angew. Chem. Int. Ed. 2013, 52, 9215-9218.

[19] S. Haubenreisser, T. H. Wöste, C. Martínez, K. Ishihara, K. Muñiz, Angew. Chem. 2016, 128, 422-426; Angew. Chem. Int. Ed. 2016, 55, 413-417.

[20] A. Sreenithya, C. Patel, C. M. Hadad, R. B. Sunoj, ACS Catal. 2017, 7, 4189-4196.

[21] a) L. Pouységu, T. Sylla, T. Garnier, L. B. Rojas, J. Charris, D. Deffieux, S. Quideau, Tetrahedron 2010, 66, 5908-5917, b) Y. Kita, T. Dohi, Chem. Rec. 2015, 15, 886-906; c) Asymmetric Dearomatization Reactions (Ed.: S.-L. You), Wiley-VCH Verlag GmbH \& Co. KGaA, Weinheim, Germany, 2016.

[22] T. Dohi, A. Maruyama, N. Takenaga, K. Senami, Y. Minamitsuji, H. Fujioka, S. B. Caemmerer, Y. Kita, Angew. Chem. 2008, 20, 3847-3850; Angew. Chem. Int. Ed. 2008, 47, 3787-3790.

[23] T. Dohi, N. Takenaga, T. Nakae, Y. Toyoda, M. Yamasaki, M. Shiro, H. Fujioka, A. Maruyama, Y. Kita, J. Am. Chem. Soc. 2013, 135, 4558-4566.

[24] M. Uyanik, T. Yasui, K. Ishihara, J. Org. Chem. 2017, 82, 11946-11953.

[25] S. J. Murray, H. Müller-Bunz, H. Ibrahim, Chem. Commun. 2012, 48, 6268-6270.

[26] M. Bekkaye, G. Masson, Synthesis 2016, 48, 302-312.

[27] N. Jain, S. Xu, M. A. Ciufolini, Chem. Eur. J. 2017, 23, 4542-4546.

[28] M. Uyanik, N. Sasakura, M. Mizuno, K. Ishihara, ACS Catal. 2017, 7, 872-876.

[29] D. Y. Zhang, L. Xu, H. Wu, L. Z. Gong, Chem. Eur. J. 2015, 21, 10314-10317.

[30] A. M. Harned, Tetrahedron Lett. 2014, 55, 4681-4689.

[31] K. Muñiz, L. Fra, Synthesis 2017, 49, 2901-2906.
[32] T. Hashimoto, Y. Shimazaki, Y. Omatsu, K. Maruoka, Angew. Chemie 2018, DOI 10.1002/ange.201803889.

[33] M. Uyanik, T. Yasui, K. Ishihara, Bioorg. Med. Chem. Lett. 2009, 19, 3848-3851.

[34] a) D.-Q. Dong, S.-H. Hao, Z.-L. Wang, C. Chen, Org. Biomol. Chem. 2014, 12, 4278-4289; b) E. Merritt, B. Olofsson, Synthesis 2011, 2011, 517-538; c) B. Basdevant, A.-A. Guilbault, S. Beaulieu, A. J.-D. Lauriers, C. Y. Legault, Pure Appl. Chem. 2017, 89, 781-789.

[35] a) B. C. Schardt, C. L. Hill, Inorg. Chem. 1983, 22, 1563-1565; b) R. M. Moriarty, H. Hu, Tetrahedron Lett. 1981, 22, 2747-2750; c) G. F. Koser, A. G. Relenyi, A. N. Kalos, L. Rebrovic, R. H. Wettach, J. Org. Chem. 1982, 47, 2487-2489.

[36] A. Jobin-Des Lauriers, C. Y. Legault, Org. Lett. 2016, $18,108-111$.

[37] J. A. Souto, C. Martínez, I. Velilla, K. Muñiz, Angew. Chem. 2013, 4, 1363-1367; Angew. Chem. Int. Ed. 2013, 52, 1324-1328.

[38] R. K. Pluta, P. E. Krach, L. Cavallo, L. Falivene, M. Rueping, ACS Catal. 2018, 8, 2582-2588.

[39] a) T. Wirth, U. H. Hirt, Tetrahedron: Asymmetry 1997, 8, 23-26; b) T. Wirth, J. Org. Chem. 1998, 63, 76747679 ; c) U. H. Hirt, M. F. H. Schuster, A. N. French, O. G. Wiest, T. Wirth, Eur. J. Org. Chem. 2001, 2001, 1569-1579.

[40] U. Farooq, S. Schäfer, A. U. H. A. Shah, D. M. Freudendahl, T. Wirth, Synthesis 2010, 6, 1023-1029.

[41] J. Yu, J. Cui, X.-S. Hou, S.-S. Liu, W.-C. Gao, S. Jiang, J. Tian, C. Zhang, Tetrahedron: Asymmetry 2011, 22, 2039-2055.

[42] A.-A. Guilbault, C. Y. Legault, ACS Catal. 2012, 2, 219-222.

[43] A. A. Guilbault, B. Basdevant, V. Wanie, C. Y. Legault, J. Org. Chem. 2012, 77, 11283-11295.

[44] a) P.-O. Norrby, T. B. Petersen, M. Bielawski, B. Olofsson, Chem. Eur. J. 2010, 16, 8251-8254; b) E. Stridfeldt, E. Lindstedt, M. Reitti, J. Blid, P.-O. Norrby, B. Olofsson, Chem. Eur. J. 2017, 23, 1324913258.

[45] S. Arava, J. N. Kumar, S. Maksymenko, M. A. Iron, K. N. Parida, P. Fristrup, A. M. Szpilman, Angew. Chem. 2017, 129, 2643-2647; Angew. Chem. Int. Ed. 2017, $56,2599-2603$.

[46] G. F. Koser, in Hypervalent Iodine Chemistry, (Ed.: T. Wirth), Springer, Berlin, 2003; pp. 137-172.

[47] B. Basdevant, C. Y. Legault, Org. Lett. 2015, 17, 49184921.

[48] a) Y. Yamamoto, H. Togo, Synlett 2006, 37, 798-800; b) J. Akiike, Y. Yamamoto, H. Togo, Synlett 2007, 39, 2168-2172. 
[49] G. Levitre, A. Dumoulin, P. Retailleau, A. Panossian, F. R. Leroux, G. Masson, J. Org. Chem. 2017, 82, 11877-11883.

[50] S. Beaulieu, C. Y. Legault, Chem. Eur. J. 2015, 21, 11206-11211.

[51] S. Suzuki, T. Kamo, K. Fukushi, T. Hiramatsu, E. Tokunaga, T. Dohi, Y. Kita, N. Shibata, Chem. Sci. 2014, 5, 2754-2760.

[52] H. Wu, Y. P. He, L. Xu, D. Y. Zhang, L. Z. Gong, Angew. Chem. 2014, 126, 3534-3537; Angew. Chem. Int. Ed. 2014, 53, 3466-3469.

[53] J. Wang, Y. Yuan, R. Xiong, D. Zhang-Negrerie, Y. Du, K. Zhao, Org. Lett. 2012, 14, 2210-2213.

[54] M. Fujita, Tetrahedron Lett. 2017, 58, 4409-4419.

[55] A. Alhalib, S. Kamouka, W. J. Moran, Org. Lett. 2015, 17, 1453-1456.

[56] C. Gelis, A. Dumoulin, M. Bekkaye, L. Neuville, G. Masson, Org. Lett. 2017, 19, 278-281.

[57] U. Farid, T. Wirth, Angew. Chem. 2012, 124, 35183522; Angew. Chem. Int. Ed., 2012, 51, 3462-3465.

[58] P. Mizar, A. Laverny, M. El-Sherbini, U. Farid, M. Brown, F. Malmedy, T. Wirth, Chem. Eur. J. 2014, 20, 9910-9913.

[59] K. Aertker, R. J. Rama, J. Opalach, K. Muñiz, $A d v$. Synth. Catal. 2017, 359, 1290-1294.

[60] Q. Wang, W. Zhong, X. Wei, M. Ning, X. Meng, Z. Li, Org. Biomol. Chem. 2012, 10, 8566-8569.

[61] W. Kong, P. Feige, T. de Haro, C. Nevado, Angew. Chem. 2013, 9, 2529-2473; Angew. Chem. Int. Ed. 2013, 52, 2469-2473.

[62] K. M. Mennie, S. M. Banik, E. C. Reichert, E. N. Jacobsen, J. Am. Chem. Soc. 2018, 140, 4797-4802.

[63] E. M. Woerly, S. M. Banik, E. N. Jacobsen, J. Am. Chem. Soc. 2016, 138, 13858-13861.

[64] Y.-B. Kang, L. H. Gade, J. Am. Chem. Soc. 2011, 133, 3658-3661.

[65] T. H. Wöste, K. Muñiz, Synthesis 2016, 48, 816-827.

[66] I. G. Molnár, R. Gilmour, J. Am. Chem. Soc. 2016, 138, 5004-5007.

[67] I. G. Molnár, C. Thiehoff, M. C. Holland, R. Gilmour, ACS Catal. 2016, 6, 7167-7173.

[68] a) S. M. Banik, J. W. Medley, E. N. Jacobsen, J. Am. Chem. Soc. 2016, 138, 5000-5003; b) S. M. Banik, J. W. Medley, E. N. Jacobsen, Science 2016, 353, 5154.

[69] U. Farid, F. Malmedy, R. Claveau, L. Albers, T. Wirth, Angew. Chem. 2013, 125, 7156-7160; Angew. Chem. Int. Ed. 2013, 52, 7018-7022.

[70] a) F. Malmedy, T. Wirth, Chem. Eur. J. 2016, 22,
16072-16077; b) M. Brown, R. Kumar, J. Rehbein, T. Wirth, Chem. Eur. J. 2016, 22, 4030-4035.

[71] J. Qurban, M. Elsherbini, T. Wirth, J. Org. Chem. 2017, 82, 11872-11876.

[72] a) A. Lishchynskyi, K. Muñiz, Chem. Eur. J. 2012, 18, 2212-2216; b) J. A. Souto, Y. González, A. Iglesias, D. Zian, A. Lishchynskyi, K. Muñiz, Chem. Asian J. 2012, 7, 1103-1111.

[73] C. Röben, J. A. Souto, Y. González, A. Lishchynskyi, K. Muñiz, Angew. Chem. 2011, 123, 9478-9482; Angew. Chem. Int. Ed. 2011, 50, 9478-9482. 


\section{REVIEW}

Enantioselective Iodine(I/III) Catalysis in Organic Synthesis

Adv. Synth. Catal. Year, Volume, Page - Page

Andrea Flores, Eric Cots, Julien Bergès, and Kilian Muñiz*

From chiral catalyst design...

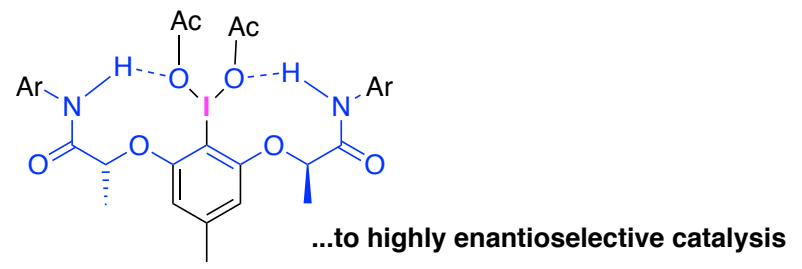

chiral supramolecular

AcO

scaffold

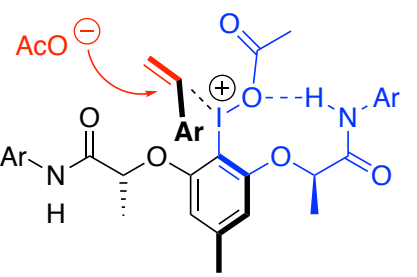

